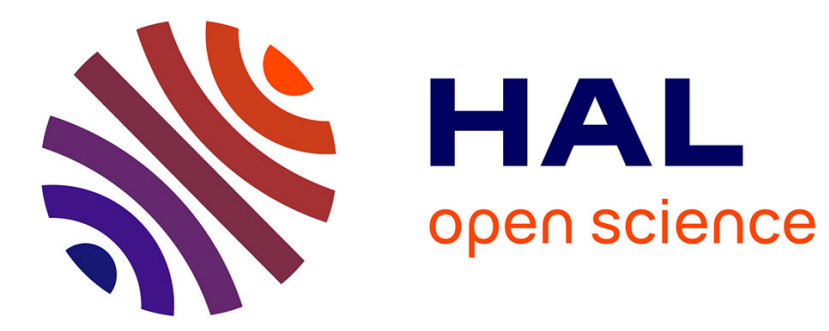

\title{
Les milliaires ardéchois d'Antonin le Pieux
}

\author{
Joëlle Napoli, René Rebuffat
}

\section{To cite this version:}

Joëlle Napoli, René Rebuffat. Les milliaires ardéchois d'Antonin le Pieux. Gallia - Fouilles et monuments archéologiques en France métropolitaine, 1992, 49, pp.51-79. 10.3406/galia.1992.2929 . hal01912696

\section{HAL Id: hal-01912696 \\ https://hal.science/hal-01912696}

Submitted on 19 Jan 2020

HAL is a multi-disciplinary open access archive for the deposit and dissemination of scientific research documents, whether they are published or not. The documents may come from teaching and research institutions in France or abroad, or from public or private research centers.
L'archive ouverte pluridisciplinaire HAL, est destinée au dépôt et à la diffusion de documents scientifiques de niveau recherche, publiés ou non, émanant des établissements d'enseignement et de recherche français ou étrangers, des laboratoires publics ou privés.

\section{(이) $\$$}

Distributed under a Creative Commons Attribution - NonCommercial - NoDerivatives| 4.0 


\title{
Les milliaires ardéchois d'Antonin le Pieux
}

\author{
par Joëlle NAPOLI et René REBUFFAT*
}

Les dix-huit milliaires connus de la cité des Helviens, posés sous Antonin, du Rhône à Alba et d'Alba à a trontière des Arécomiques, permetlent de faire une étude comparative. Les inscriptions nécessaires, une civiquantaine, étaient exécutées (en deux étapes) par deux ateliers; la limite de leur intervention était probablen⿳⺈⿴囗十⺝ de certains postes, et montrer que ce système de répartition du travail se rencontre ailleurs, en particulier sur la vie domitienne. Des descriptions plus minutieuses des milliaires connus permettraient certainement d'étendre ce travail à d'autres voies. On a pu également ajouter quelques précisions à la description des monuments.

The 18 milestones known from the Roman city of Alba Heluiorum were placed under Antoninus Pius from the Rhone river to Alba in the east and extended from Alba south, to the frontier of the Volcae Arecomici. They afford the possibility of a comparative study. This fifly miles network was prepared (in two steps) by two workshops, one in the north, one in the south of a crossway (the milestone South-Ten). It is not only possible to determine the exact location of several milestones, but also to distinguish the workshops' styles and how the labour forces were organized to accomplish the project. The different workshops' styles are particularly evident on the Via Domitiana. Furthermore, this study includes delailed description, which should facilitate the study of the other Roman roads.

Mots clés : ateliers, commanditaires, épigraphie, gravure, milliaires, ordinatio, voie romaine, Alba, Antonin, Ardèche, Helviens, voie domitienne.

* C.NRS. LMR 126-2, Groupe de recherches sur l'armée romaine et les provinces. 
Au cours d'un séminaire sur les milliaires, la documentation fournie par le département de l'Ardèche avait retenu notre attention. Or toutes les circonstances ont favorisé une étude : la permission d'enquêter sur place, aimablement accordée pendant deux années consécutives, par M. J. Lasfargues, Directeur des Antiquités historiques de la région Rhône-Alpes; l'aimable accueil que nous avons reçu dans le département de l'Ardèche ${ }^{1}$, auprès de diverses institutions ${ }^{2}$ et de nombreux particuliers ${ }^{3}$;

\section{Ouvrages cités en abrégé}

\section{Carte archéologique ou BLANC}

Bt.anc A., Carte archéologique de la Gaule romaine, fasc. XV, Ardèche, Paris, Éd. C.NRS, 1975, 100 p., 8 pl.

CIL XVII, 2

Corpus Inscriptionum Latinarum, 1986.

Cf. nos remarques sur cet ouvrage, infra, p. 75-76.

ESP. ou EsPÉRANDIEU

Espérandieu E., Inscriptions latines de Gaule Narbonnaise (ILGN), Paris, 1929. le L31.

Cet ouvrage donne sous le $n^{\circ} 653$ le L21 et sous le $n^{\circ} 652$

Histoire du Languedoc

IIistoire générale du Languedoc, XV, 1892-1893, Toulouse.

Cet ouvrage comporte un numéro 39 qui ne figure pas dans nos tableaux et qui désigne les milliaires signalés par $\mathrm{J}$. Oll.hí 1): Маніснан), Revue épigraphique du Midi de la France, I, p. 45, no 66 . Il s'agit de milliaires d'Antonin, mais dont on ne sait s'ils font ou non double emploi avec les autres (voir Lauxerois sous le $n^{\circ} 39$ ).

L'ouvrage ne connait pas le milliaire L21 de Cruas qui a été trouvé en 1925 et le milliaire L31 dit de Mirabel qui a èté trouvé en 1897.

KÖNIG, 1970

KöNIG I., Die Meilensteine der Gallia Narbonensis, Studien zum Strassenwesen der Provincia Narbonensis, Berne, 1970.

LAUXEROIS, 1983

Lauxerois R., Le Bas Vivarais à l'époque romaine. Recherches sur la cité d'Alba, 9e suppl. à la Revue archéologique de Narbonnaise, Paris, 1983, 320 p., 11 fig., 7 pl.

Des bibliographies très larges sont données par Blanc, Lauxerois et le CIL XII et. XVII, 2.

I M. R. Chapuis, député-maire du Teil, a chaleureusement accueilli notre projet (si même il ne l'a pas quelque peu suscité) et nous avons en toute occasion trouvé informations et encouragements à la mairie du Teil auprès de lui-même et de ses collaborateurs.

2 Nous avons été aimablement renseignés par D. Dupraz, Directeur des archives départementales de Privas. M. l'intendant et le personnel du lycée de Privas ont facilité l'existence de la Carte archéologique et d'un excellent inventaire commenté, donné par Roger Lauxerois ${ }^{4}$ dans son ouvrage sur Le Bas-Vivarais à l'époque romaine 5 .

Pourquoi cette enquête? Une voie romaine propre au territoire des Helviens a été bornée sous Antonin le Pieux, et par chance, une partie de ces milliaires est conservée, une autre est connue par des descriptions assez précises. Or nous recherchions une série de milliaires mise en place en une seule opération (cf. infra, p. 63, fig. 13).

Ce qui nous intéressait, dans un cas comme celui-là, n'était pas le dossier des textes. Ce dossier était connu et largement commenté. Nous voulions savoir si, à partir d'une série cohérente et accessible, des informations pouvaient être obtenues sur les étapes de la fabrication d'une série de milliaires.

Un travail parallèle sur d'autres séries publiées avec assez de précision ${ }^{6}$ pour fournir matière à l'étude nous montrait que ce travail était possible, pourvu qu'on s'attachât aux plus minimes particularités. Il faut bien dire que dans beaucoup de cas, nos

notre examen du milliaire conservé dans le jardin du lycée; $\mathrm{M}^{\text {me }}$ la conservatrice du musée de Vagnas nous a accueillis quand nous avons examiné les fragments du milliaire; nous avons consacré deux visites successives au musée de Nîmes, pour examiner le milliaire de Barjac; de nombreuses personnes, à la mairie, au syndicat d'initiative, nous ont aidés à chercher la clé de la baraque de chantier de Cruas, pour laquelle nous avons également dérangé $M$. Flavigny, architecte des Monuments historiques de l'Ardèche et de la Drôme : nous remercions $M$. le curé de Cruas d'avoir bien voulu nous la procurer. $M^{\text {me }}$ F. Beck nous a accueillis au musée de Saint-Germain-en-Laye, où est conservé le milliaire de Mirabel, et nous a donné une copie du dossier inédit qui le concernait.

3 Nous avons dérangé deux fois de suite la propriétaire de la villa dont l'allée débouche auprès du milliaire de SaintGermain-d'Ardèche; nous avons travaillé toute une après-midi dans le jardin de $\mathbf{M}$. et $\mathbf{M}^{\mathrm{me}}$ Vallentin du Cheylard, à Montélimar, et c'est avec la plus grande tristesse que nous pensons que M. Vallentin du Cheylard ne pourra plus lire le résultat de notre enquête.

4 Dans tout le cours de notre exposé, les chiffres arabes, de 20 à 43 (éventuellement précédés de L : L 20), sont les numéros de milliaires du catalogue de R. Lauxerois. Les chiffres romains sont ceux des milles pas.

5 A. Blanc, Carle archéologique de la Gaule romaine, fasc. XV, Ardèche, Paris, C.NRS, 1975, avec p. 31-33 l'essentiel des remarques que suscitent les milliaires; - R. LauxeRoIs, Le Bas Vivarais à l'époque romaine, Recherches sur la cité d'Alba, Paris, 1983. Nous remercions M. Lauxerois de son accueil au musée de Vienne, où nous l'avons informé des raisons et du sujet particulier de notre enquête.

6 I. Könı, Die Meilensteine der Gallia Narbonensis, Itinera Romana 3, Berne, 1970. 
catalogues épigraphiques interdisent une pareille étude: ils abondent même en rubriques qui se réduisent à un "mème texte", ce qui peut satisfaire l'historien, voire l'historien de la route, mais non l'historien des milliaires.

Ce laconisme n'est pas, fort heureusement, le cas du catalogue des milliaires dressé par R. Lauxerois. Mais il pouvait cependant se faire qu'au fil des comparaisons, les minimes détails dont nous parlions méritassent d'être vérifiés. Nous avons donc examiné tous les milliaires qui nous étaient accessibles, en nous astreignant à les dessiner intégralement, et nous donnons sous forme de tableaux les résultats de nos observations.

A l'issue de cette étude, nous pouvons avancer une théorie sur les étapes de fabrication d'une série donnée de milliaires, essayer de la comparer avec la genèse d'autres séries, et adresser quelques supplications précises aux épigraphistes qui publient des milliaires (voir infra, p. 68).
Quelques mots de présentation des milliaires de la voie des Helviens sont nécessaires. Elle mérite ce nom, car le bornage s'arrête tout net à la limite sud de l'ancienne cité, à la frontière des Volques Arécomiques de Nîmes ${ }^{7}$; il en était très probablement de même au nord, à la limite des Ségovellauniens de la cité de Valence ${ }^{8}$. La voie va du Rhône à $A l b a$

7 Lauxerois, 1983. p. 56-58.

8 A. Blanc (1975) place sur la carte (p. 32) la frontière nord entre l'Ouvèze et la Payre et pense à la vallée de la Payre p. 33 ; - R. Lauxerois (1983, p. 59-60) rassemble la documentation connue et conclut avec prudence a... nous sommes bien incapables de fixer le trace d'une frontièren, mais avance des suggestions intéressantes : "La montagne de Rompon, en face du confluent de la Dròme et du Rhòne, la large vallée de la Payre, ou l'interfluve entre Ouvèze et Payre constituent autant de régions naturelles qui pouvaient s'imposer comme zone de démarcation". Vous avons retenu la première de ces hypothèses dans notre annexe II, infra, p. 78, en fonclion de notre hypothèse de travail sur les milliaires.

\section{Famille Nord}

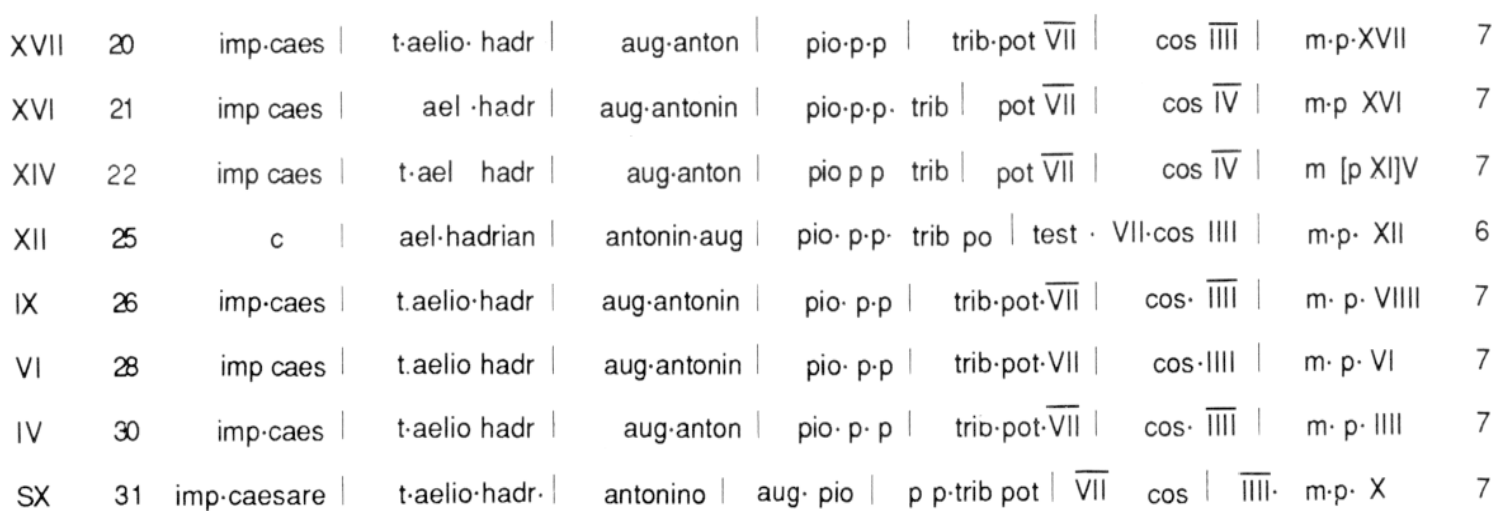

Famille Sud

\begin{tabular}{|c|c|c|c|c|c|c|c|c|c|}
\hline XIII & 32 & imp caesar & tito ael ha & $\mathrm{dr}$ antoni & \multicolumn{2}{|c|}{ no aug pio } & $p p$ trib pot & VII $\cos \| I I$ & $m p$ \\
\hline XVII & 33 & imp tito & aelio ha & drian ano & tonino & aug pio & $p p$ trib po & VII $\cos \| I I$ & m p XVII \\
\hline$X X$ & 34 & imp tito & aelio ha & $\mathrm{dr}$ anto & nino [aug] & pio $p p$ trib & pot VII & $\cos \|I\|$ & $m p X X$ \\
\hline$X X I$ & 36 & imp tito & aelio ha & drian an & tonino & aug pio & $p p$ trib pot & VII $\cos \|I\|$ & $m p X X I$ \\
\hline$X X I 1$ & 36 & imp tito & aelio ha & drian an & tonino & aug pio & $p p$ trib pot & VII $\cos \|I\|$ & $m p X X I I$ \\
\hline XXIII & 37 & & & & & & $p p$ trib pot & VII $\cos$ IIII & $m p X X I I I$ \\
\hline XXIV & 38 & imp tito & aelio ha & drian an & tonino & aug pio $p p$ & trib pot & VII $\cos \| I I$ & \\
\hline$X X X$ & 41 & & & & $\operatorname{nin}[0]$ & aug pio & $p p$ trib pot & VII cos IV & $m p X X X$ \\
\hline$X X X I$ & 42 & imp.t ael & hadria & no anto & nino aug & pio $p p$ trib & pot VII & c & $X X X I$ \\
\hline$X X X I I I$ & 43 & & & & no aug $\mid$ & piopp & trib pot & VII cos IV & $m p X X X \mid I$ \\
\hline
\end{tabular}


Helviorum, et d'Alba à la frontière arécomique. Le bornage a certainement été commandé et payé par la cité des Helviens, ce qui montre, soit dit en passant, que les bornages n'étaient pas toujours des œuvres nécessaires au peuple romain, ou à la gloire de l'empereur régnant, mais qu'ils pouvaient être considérés comme utiles par une collectivité locale, que cette utilité soit pratique, de prestige, ou plus complexe.

Ces milliaires d'une même année se ressemblent de facture, comme nous allons le dire en y revenant plus précisément. Mais ils comportent pour leur début des textes différents, soit, en lecture intégralement développée (qui ne se présente jamais sur la pierre) :

$$
\begin{array}{cc}
\text { I - imperalore caesare } & \text { tito aelio hadriano augusto } \\
\text { II - imperatore } & \begin{array}{l}
\text { antonino ... } \\
\text { lito aelio hadriano antonino } \\
\text { augusto ... }
\end{array} \\
\text { III - imperatore caesare lilo aelio hadriano antonino } \\
\text { augusto ... }
\end{array}
$$

En revanche, la suite du texte est identique sur les milliaires, et ce ne sont plus que ses versions qui varient :

... pio p p trib pot (ou polest) vii cos iiii (ou iv) $m p$... suit le chiffre des milles, comptés à partir de la cité d'Alba.

Avant de mesurer la portée de ces différences, nous devons préciser notre vocabulaire.

\section{Vocabulaire}

Bloc: le parallélépipède extrait de la carrière.

Support: le milliaire taillé, mais non inscrit, ou considéré sans son inscription. Se subdivise en base et fût.

Milliaire : le milliaire une fois inscrit.

Texte : le texte à inscrire, abstraction faite des abréviations et des alinéas.

Disposition : disposition du texte en alinéas successifs.

Version: le texte inscrit, abrégé et disposé. Nous n'avons pas besoin ici d'un mot particulier pour le texte abrégé, mais non disposé.

Ordinatio: composition de la version du texte.

Ordonnateur : auteur de cette composition (nous évitons le mot ordinateur pour des raisons faciles à comprendre).

\section{Conventions}

Interponctions : représentées par un point ordinaire (nos machines ne comportaient pas de signe convenable en milieu de ligne).
Majuscules : les majuscules initiales du français sont strictement exclues des initiales de mots latins : imp caes $t$ aelius hadrianus, etc.

De : lieu de trouvaille : "De Meysse ...."

$A$ : lieu de conservation : "... à Montélimar".

\section{LES MILLIAIRES}

\section{DE LA ROUTE DES HELVIENS}

Au nord d'Alba: N I à XVII (plus quelques-uns) Au sud d'Alba : S I à XXXIII

Total : 50 milliaires au moins.

\section{Les douze milliaires conservés}

20 N XVII De Cruas, près de l'église de Cruas, vers l'ouest (fig. 1)

21 N XVI De Cruas, près de l'église de Cruas, vers l'est (fig. 2)

22 N XIV Du Ferrand, baraque de chantier de Cruas

$25 N$ XII De Meysse, collection Vallentin du Cheylard à Montélimar (fig. 3)

26 N IX De Rochemaure, dans la cour du lycée de Privas (fig. 4)

30 N IV De la route et sur la route $\mathrm{N} 540 \mathrm{du}$ Teil à Alba (fig. 5)

31 S X De Mirabel, musée de Saint-Germain-enLaye (fig. 6)

$32 \mathrm{~S}$ XII/XIII De et à Saint-Germain-d'Ardèche, calvaire (fig. 7)

34 S XX De et à Pradons, sur la N 579, calvaire (fig. 8)

$41 \mathrm{~S} X X X$ De et à Salavas, voisinage de la mairie (fig. 9)

42 S XXXI De et à Vagnas, N 579, calvaire; fragments au musée de Vagnas (fig. 10 et 11)

$43 \mathrm{~S}$ XXXIII De Barjac, au musée archéologique de Nîmes (fig. 12).

\section{Les six milliaires connus et perdus}

28 N VI Environs du Teil ou de Rochemaure 33 S XVII Entre Saint-Maurice-d'Ardèche et la Borie de Balazuc

35 S XXI Entre Pradons et Ruoms

36 S XXII Ruoms, à la Croix de Ruoms

37 S XXIII Ruoms

38 S XXIIII Du sud de Ruoms. 


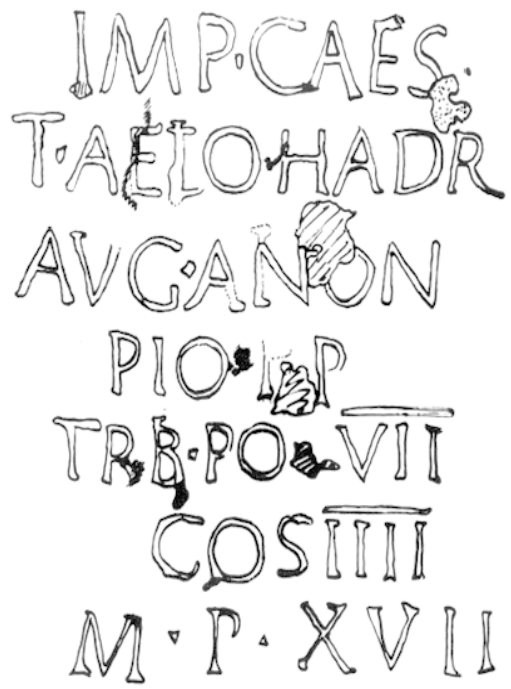

Fig. $1-20$ N XVII. A Ciruas.

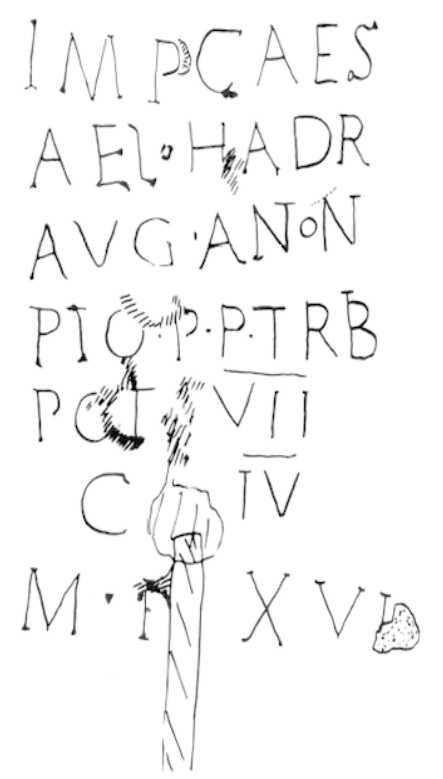

Fig. $2-21$. XVI. A Cruas.

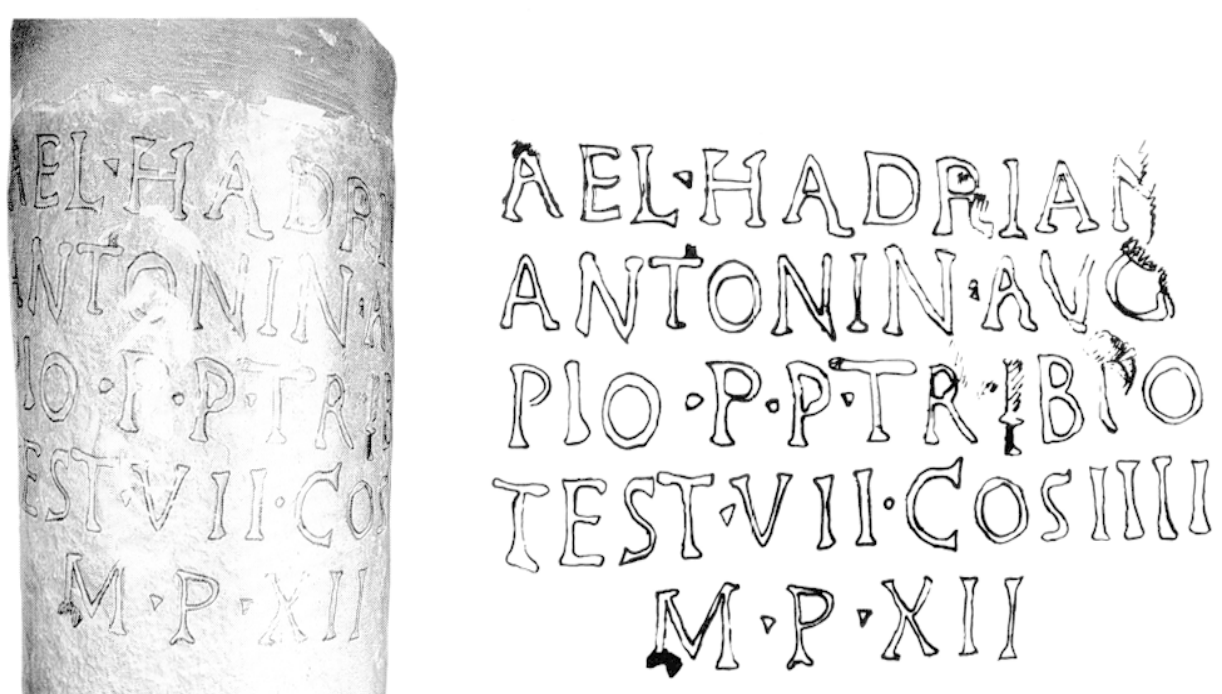

Fig. $3-25$ V XII. De Meysse, à Montélimar. 

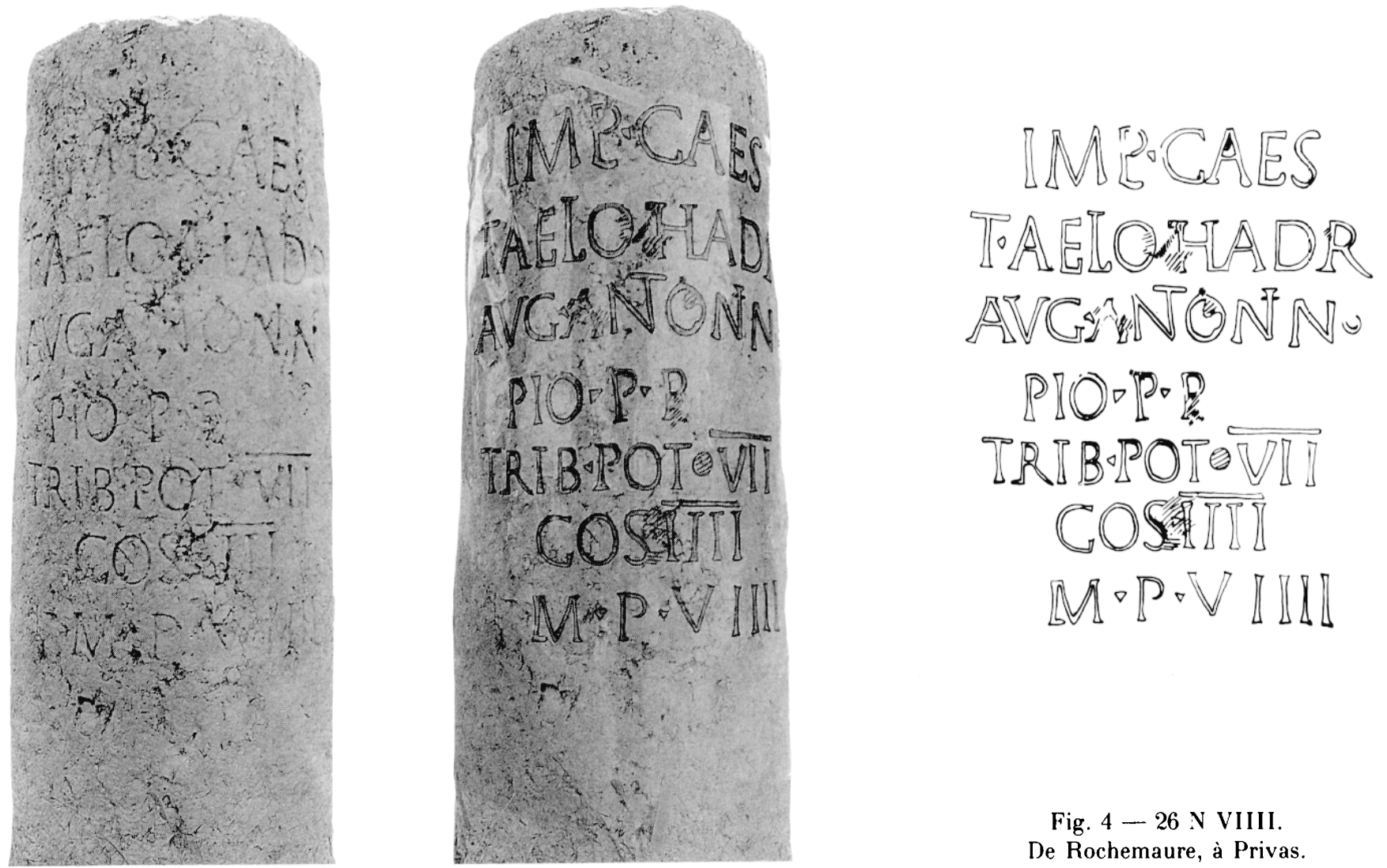

Fig. $4-26 \mathrm{~N}$ VIIII.

De Rochemaure, à Privas.
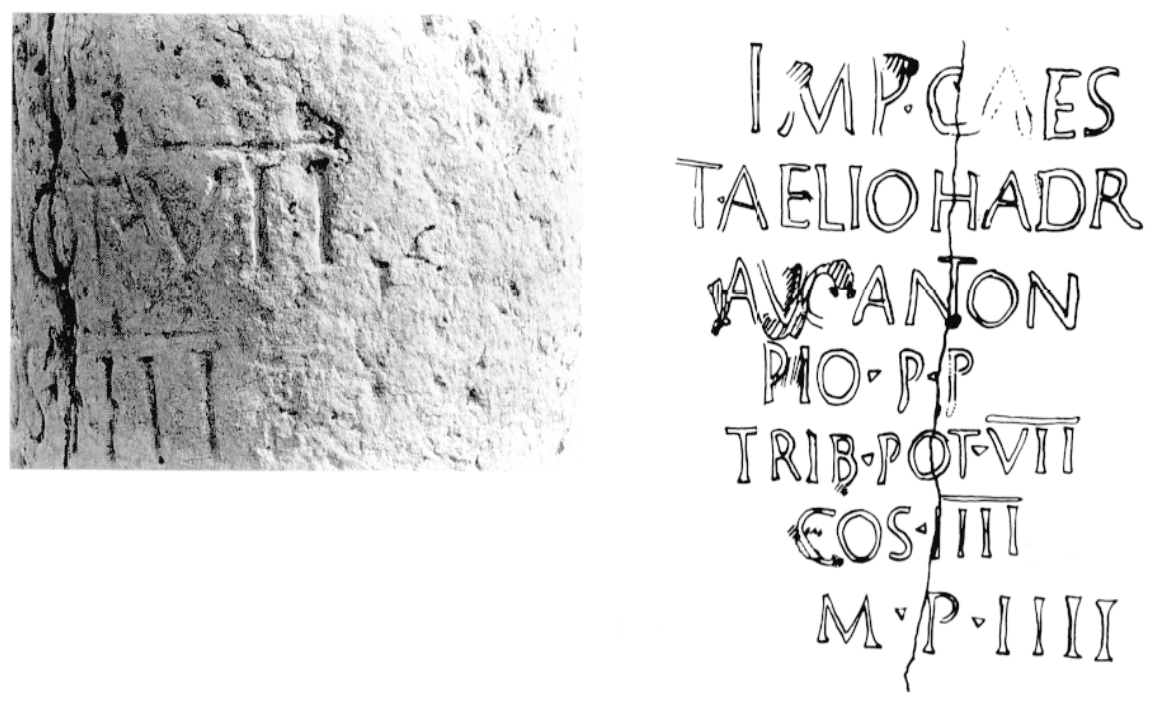

Fig. $5-30 \mathrm{~N}$ IIII. Nationale 540 du Teil à Alba. Vue d'un détail du milliaire et dessin de l'inscription. 


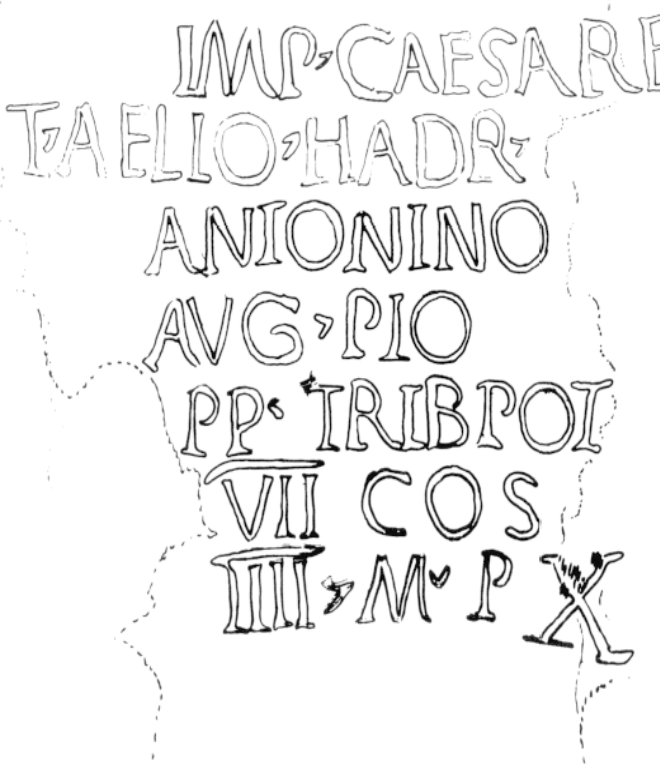

Fig. $6-31 \mathrm{~s} \mathrm{X}$.

De Mirabel, à Saint-Germain-en-Laye.

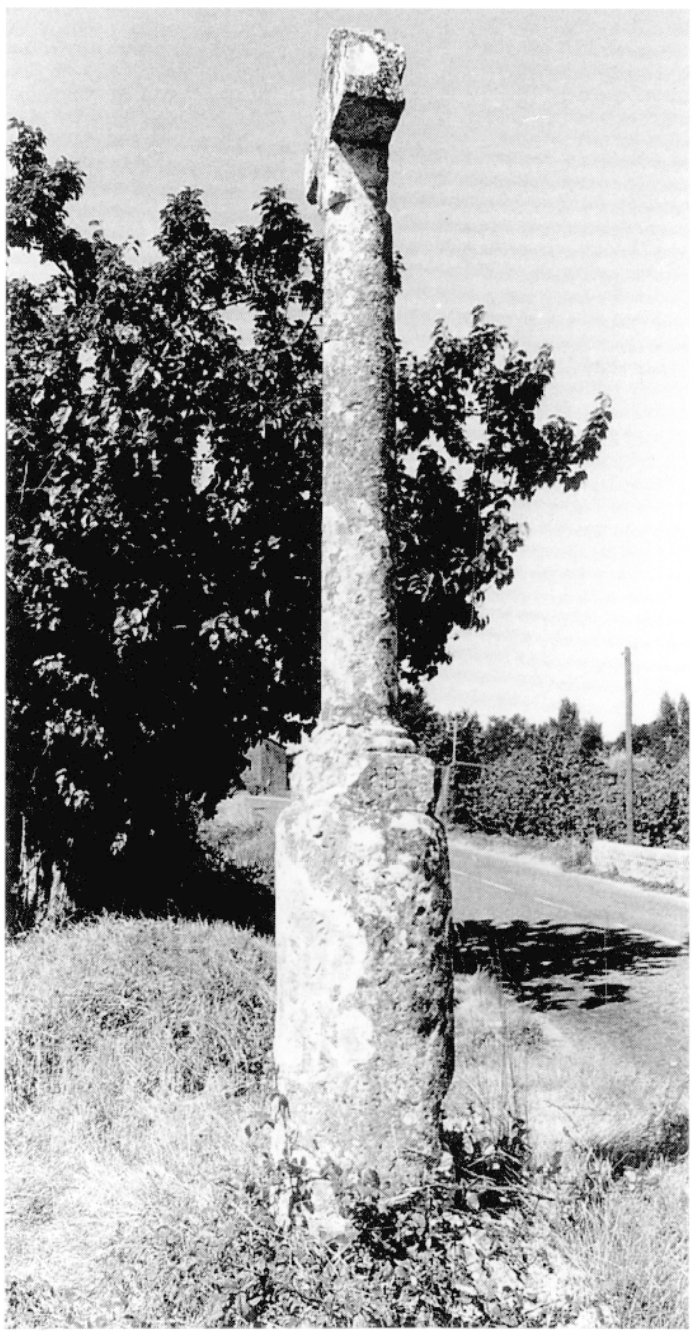

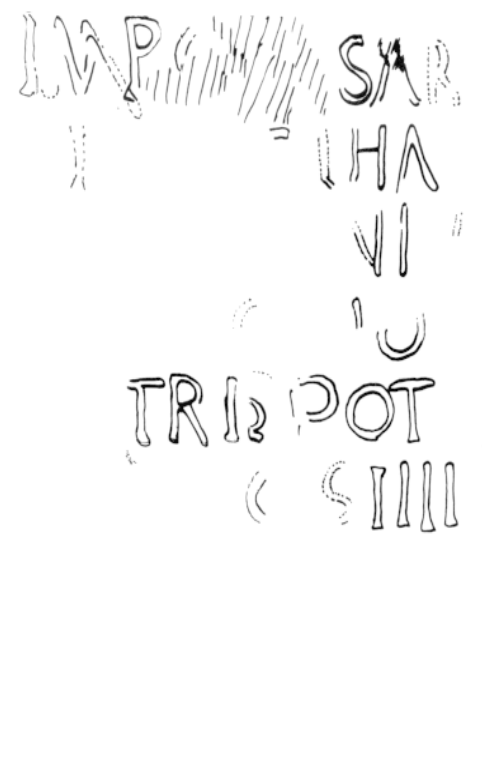

Fig. $7-32 \mathrm{~S}$ XIII.

De et à Saint-Germain-d'Ardèche.

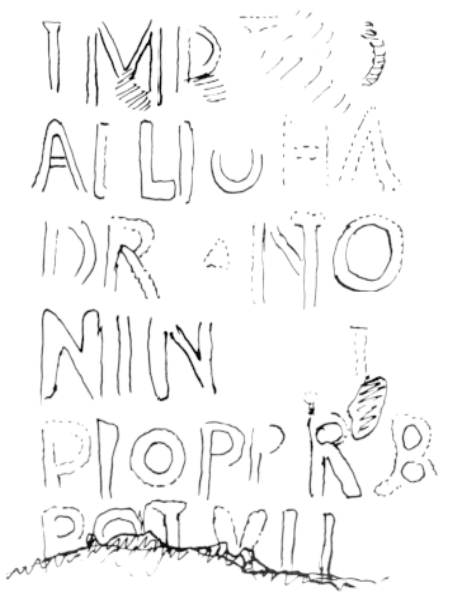

Fig. $8-34 \mathrm{~S} X \mathrm{X}$.

Calvaire de la Nationale 579, à Pradons. 


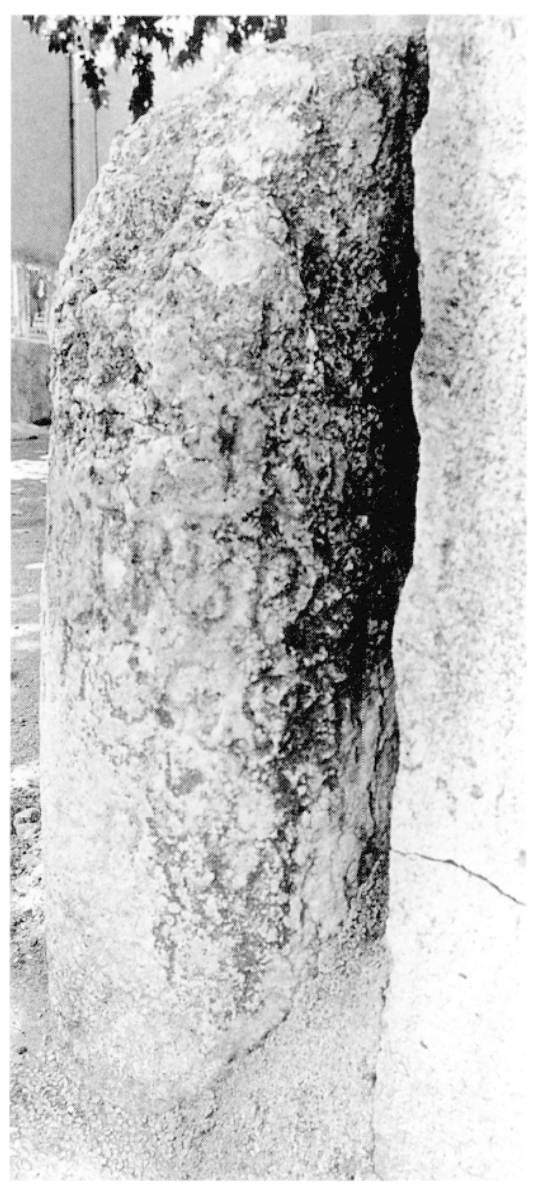

Fig. 9-41 S XXX. De et à Salavas.

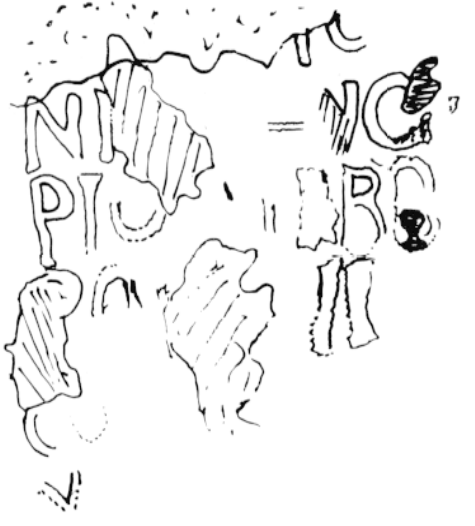

Fig. $10-42 \mathrm{~S}$ XXXI.

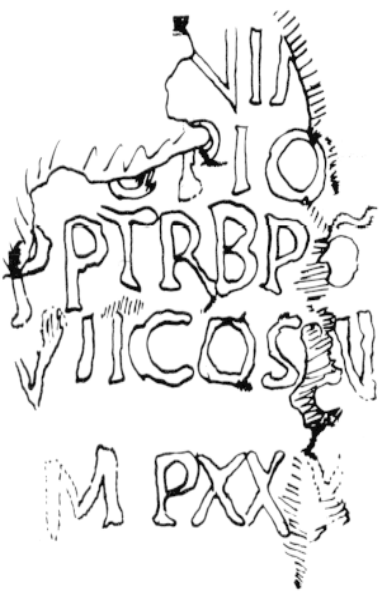

De et à Vagnas.

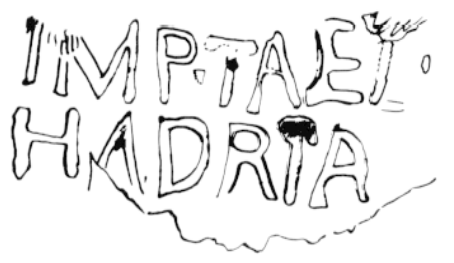

Fig. $11-42 \mathrm{~S} \times X X I$. De et à Vagnas.

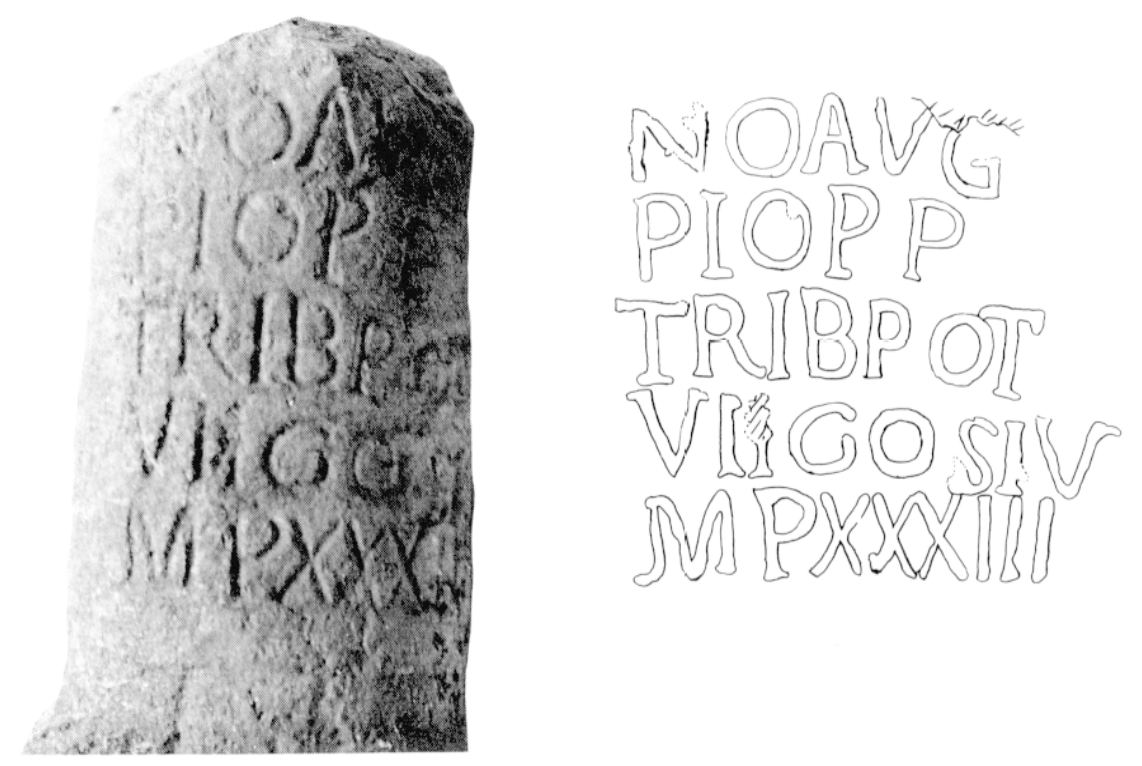

Fig. $12-43$ S XXXIII. De Barjac, à Nîmes. 


\section{Les milliaires disparus}

$\mathrm{N}$ Chiffres au-delà de XVII (voir infra, annexe II)

$\mathrm{N} X \mathrm{~V}$

$\mathrm{N}$ XIII

$\mathrm{N}$ XI, $\mathrm{X}$

$\mathrm{N}$ VIII, VII

N.V

N III, II, I

S I, II, III, IIII, V, VI, VII, VIII, IX

$\mathrm{S}$ XI, XII

S XIV, XV, XVI

S XVIII, XIX

S XXV, XXVI, XXVIII, XXIX

S XXXII

- On note que la lacune la plus importante se situe au voisinage d'Alba (12 milliaires disparus);

- 22 milliaires sont perdus au sud, 10 au nord, sans compter les $\mathrm{N}$ XVIII et suivants.

\section{LES BLOCS}

\section{Origine de la pierre}

Tous les milliaires connus sont en calcaire gris, à gros grains. La finesse du grain n'est pas constante à première vue, mais cette impression demande à être vérifiée. Si elle l'était, cela n'impliquerait pas forcément qu'on ait recouru à deux ou plusieurs carrières $^{9}$.

\section{Dimensions des blocs}

La longueur du parallélépipède tiré de la carrière est rarement connue, car on a affaire tantôt à des fùts cassés, tantôt à des bases inobservables. On note :

$31: 180 \mathrm{~cm} ; 30: 175 \mathrm{~cm}$;

$26: 174 \mathrm{~cm}$ (non vérifiable actuellement);

$33,35,36: 5$ pieds 5 pouces, $176 \mathrm{~cm}$.

9 Le L 26 de Rochemaure à Privas inclut un rostre de Bélemnite. Ce type de calcaire, de Chomérac, au nord, et de Lussas, à l'est, est pour l'essentiel constant, mais bien sùr avec des variantes de veine à veine.

D'après l'inventeur Longin Ducros, le milliaire de Mirabel, conservé à Saint-Germain, pèserait presque exactement une tonne : " $1008 \mathrm{~kg}$ ". II ne semble pas qu'il s'agisse d'une pesée sur une balance communale, mais d'une évaluation (faite par des "experts de la pierre taillée»). Son volume approximatif, pour une base de $57 \times 57 \times 53 \mathrm{~cm}$ et un fût de $127 \mathrm{~cm}$ de haut sur 52 de diamètre moyen est de $0,4373 \mathrm{~m}^{3}$ ce qui représente une densité approximative de 2,3 .
Tous les blocs devaient cependant être à peu près de cette longueur.

Nous ne disposons pour le moment que de 8 cas où le côté de ce carré est connu : il oscille entre 41 et $57 \mathrm{~cm}$.

Ce qu'on connait actuellement permet de dire que les parallélépipèdes originels étaient tous semblables, aux imprécisions près, dues à la taille (à l'«équarissage") en carrière.

Il n'est pas impossible que la commande ait porté sur des blocs de 6 pieds $(177,42 \mathrm{~cm})$, mais cela importe peu à notre sujet.

\section{LES SUPPORTS}

Les milliaires comportent un fût relié à la base par des congés, c'est-à-dire des triangles taillés dans les quatre angles. Ces triangles sont généralement obliques. Le fût semble n'être jamais strictement cylindrique, mais légèrement tronconique.

Quelques différences entre la mesure directe du diamètre, et son calcul à partir de la circonférence semblent indiquer que le fût est légèrement ovale. Cela est probablement dû à la difficulté de tailler une circonférence parfaite.

Restent épannelés la base et le dessus du fût. Il est probable que le support conserve là l'épannelage de carrière. Les hauteurs connues de fûts oscillent faiblement, entre 120 et $139 \mathrm{~cm}$ : on taillait toujours la même portion approximative du bloc, mais sans s'astreindre à une mesure ou à une proportion stricte.

On n'a pas pour le moment remarqué de différence de taille d'un support à l'autre. Le grain du calcaire a fait que cette taille a abouti à des résultats imparfaits du point de vue de la gravure des caractères, le champ présentant des lacunes et des alvéoles.

Notre tableau (infra, p. 72-74) des dimensions des supports est aussi cruellement lacunaire que celui des blocs. Pour le moment, rien ne nous permet de dire que les supports n'aient pas été préparés dans un seul atelier. Dans ce cas, il est probable que cet atelier ait dépendu directement de la carrière, d'autant que la taille d'un fût cylindrique suppose un degré de maîtrise du travail de la pierre qu'on peut attribuer aux carriers.

\section{DE LA COMMANDE À LA GRAVURE}

Les milliaires de la voie des Helviens ont fait l'objet d'une commande unique : ce n'est qu'à ce stade initial qu'a pu être donnée l'indication de la 
$7^{\mathrm{e}}$ puissance tribunicienne d'Antonin et de son 4. consulat, indication soit erronée, soit étroitement limitée dans le temps ${ }^{10}$.

L'hypothèse d'une commande unique à la carrière de tous les supports nécessaires est dans ce cas satisfaisante.

L'indication du nombre des milles n'a jamais été gravée par une autre main que celle qui a gravé le reste du texte ${ }^{11}$. On en conclut que le milliaire était intégralement préparé dans son atelier de gravure, et transporté ensuite au point d'érection (probablement marqué par un repère provisoire).

On part donc de l'hypothèse d'un ou de plusieurs ateliers de gravure qui ont reçu les supports et préparé les milliaires.

\section{ClassifiCATION DES INSCRIPTIONS}

Une première classification a été présentée par R. Lauxerois ${ }^{12}$ qui a utilisé trois critères : le texte, les abréviations, la disposition. Nous en ajoutons un quatrième : tous les milliaires ne comportent pas d'interponctions. On remarque alors aussitôt que les interponctions vont en général de pair avec le surlignage des chiffres des puissances tribuniciennes et des consulats. Ceci posé, on voit ensuite que l'interponction-surlignage est généralement caractéristique des milliaires du nord, et son absence des milliaires du sud.

\section{La famille nord}

A partir des quatre critères cités, qui sont cohérents, nous distinguons une famille nord très homogène (la série 2 de Lauxerois) : 20, 21, 22, 26, 28, 30 .

- texte : imperatore caesare tito aelio hadriano augusto anlonino;

- abréviations constantes : caes; $t$; hadr;

- abréviations flottantes : ael ou aelio; anton ou antonin, mais jamais antonino; flotte également iiii ou iv ;

10 Lauxerois, 1983, p. 126. Nous pouvons probablement éliminer l'hypothèse d'une erreur au stade de la commande pour expliquer qu'Antonin soit à la fois cos iiii et. tr $p$ vii. En effet, si faute il y avait, elle aurait été probablement corrigée quand les autres erreurs de formulation l'ont èté.

11 D'autres cas peuvent se présenter pour d'autres séries de milliaires : la gravure du nombre des milles par une autre main: l'absence d'indication du nombre de milles, et dans ce cas nous pensons que cette indication était simplement peinte. Existe-t-il des cas où les lettres MP soit de la mème main que le reste de l'inscription, et les chiffres d'une autre main, ou absents?

12 Lauxerois, 1983, p. 125-129.
- disposition : toujours sept lignes; alinéas constants, un seul flottement avant trib $(20,26,28$, 30 ) ou après $(21,22)$;

- interponctions : dans tous les cas (y compris sur le L 22 Cruas, où elles n'avaient pas été notées);

- surlignages : sauf sur le 28 , mais on est tributaire d'une ancienne lecture peut-être négligente sur ce point. A noter un hypersurlignage en $\mathrm{L} 22$ où le chiffre des milles serait surligné aussi (ce que nous n'avons pu vérifier, la pierre étant érodée).

\section{La famille sud}

Nous distinguons également une famille sud très homogène : $33,34,35,36,38$ complets ; $37,41,43$ incomplets (la série 3 de Lauxerois).

- texte : imperatore lito aelio hadriano antonino augusto ;

- non abréviations : tito; aelio ; antonino;

- abréviations flottantes: hadr ou hadrian; flotte également cos iiii ou iv;

- disposition : toujours huit lignes quand on peut l'observer; alinéas constants après tito et ha/ driano; quasi constants après pol; flottement portant sur deux à trois lettres pour antonino aug pio $p p$;

- interponctions: aucune (sauf L 38, douteuse? : c'est un milliaire perdu);

- surlignages : aucun ;

- ligature : leur étude est moins parlante que celle des autres critères. Cependant, la ligature $A N$ dans hadriAN ou dans ANtonino est propre au groupe sud.

Nous devons examiner dans ce cadre un cas particulier.

S XXXI L 42 Vagnas

- texte : sud;

- abréviations : comme la famille sud, il a antonino; fait isolé, il a hadriano en toutes lettres; comme deux milliaires de la famille nord, il a $t$ ael;

- disposition : comme la famille sud, huit lignes; les alinéas sont ceux de S XX L 34, mais il n'a pas la coupe caractéristique de la famille sud $h a /$ driano, à cause évidemment des abréviations $t$ ael au lieu de tito aelio;

- interponctions : une seule; proche de sud;

- surlignages : aucun, comme sud.

En fait, la seule différence avec la famille sud vient des abréviations t ael hadriano au lieu de tito aelio hadr, ou hadrian.

On peut considérer qu'il s'agit d'une variante par rapport à la famille sud. 


\section{Les milliaires à reclasser}

Nous désignons par $\mathrm{N}$ et $\mathrm{S}$ les milliaires situés au nord et sud d'Alba: en revanche, nous emploierons nord et sud, ou famille (groupe, série) nord et famille sud pour les deux familles dont nous venons de parler.

Trois milliaires n'entrent pas dans les deux familles : L 25, 31, 32 .

Ces trois milliaires ont été regroupés par R. Lauxerois, car ils offrent le même texte : imperatore caesare tito aelio hadriano antonino augusto; d'autre part, leur situation permettrait d'esquisser un groupe "central». Si N XII L 25 s'intercale au milieu de la série nord, S X L 31 et S XII/XIII L 32 se situent pour nous au début de la série sud. Ce qui gêne pour poursuivre dans ce sens, c'est que ces deux derniers milliaires sont les seuls à combler le vide qui sétend pour nous entre le mille $\mathrm{N}$ IV et le mille S XVII.

Remarquons que le texte qui est commun aux trois milliaires est correct, alors que celui du groupe nord place, à tort, augusto avant antonino, et que celui du groupe sud omet, à tort, caesare.

Il faut reprendre l'examen de chaque cas.

\section{N XII L 25 De Meysse à Montélimar}

- abréviations: ael, antonin comme nord; hadrian comme sud; potest original ;

- disposition : originale, car seule à avoir six lignes. Alinéas très voisins de nord pour les trois premières lignes; les lignes 4 et 5 répondent aux lignes 4-5-6 de nord, malgré les trois lettres supplémentaires potest.

- interponctions : très voisines de nord;

- surlignages : aucun.

Ce texte "central» a été transcrit de façon originale par rapport à nord : hadrian, potest, six lignes, pas de surlignages.

D'après la position de la lettre $\mathrm{C}$ de la première ligne, qui est encore visible, il ne serait pas impossible qu'il ait eu caesar ou caesare, à la différence de nord, qui a caes.

Le texte diffère de sud sur un point important, la présence de caesar, que sud ignore. Les abréviations diffèrent de celles du sud, car il n'a pas aelio, régulier au sud, irrégulier au nord, ni antonino, régulier au sud, inconnu au nord. La disposition en six lignes n'a aucun point commun avec celle de sud.

Ce milliaire n'a donc pratiquement rien de commun avec sud, sauf, accidentellement, peut-on penser, l'abréviation hadrian. En revanche, il est fortement apparenté à la famille nord, et on peut le considérer comme une variante de nord.
S XII/XIII L 32 De et à Saint-Germain-d'Ardèche

- abréviations : caesar proche de L 31 caesare et L 25; lito, antonino comme sud; ael hadr comme nord ;

- disposition : sept lignes, comme nord; coupe ha/dr comme sud; lignes $4,5,6,7$ comme sud antoni/no ne se trouve pas ailleurs;

- interponctions : aucune;

- surlignages : aucun.

Avec un mot de plus, la version rattrape la disposition de sud avec la coupe $\mathrm{HA}$ / à la deuxième ligne; la disposition ultérieure n'est guère différente de sud.

On a donc l'impression qu'on a affaire à une version sud, corrigée par l'adjonction du mot caesar.

S X L 31 De Mirabel à Saint-Germain-en-Laye

- abréviations: caesare, original; antonino, comme sud; $t$ aelio hadr, comme nord;

- disposition : sept lignes, comme nord; chiffre des milles non isolé, ce qui est original ; les deux premières lignes, disposées comme nord ; les lignes 3-4-5 à la manière de sud;

- interponctions : à la manière de nord;

- surlignages : oui, comme nord.

Nous n'avons ici qu'une relation avec sud qui soit précise, c'est antonino en toutes lettres. Tout le reste rappelle nord. Sont originaux le texte, caesare et la dernière ligne.

Si nous faisons l'hypothèse que le texte ait été préparé à partir de la version nord, qu'il suit pour les deux premières lignes, la modification était provoquée par l'adoption du texte correct antonino augusto au lieu de augusto antonino. D'autre part, caesare, aelio, antonino en toutes lettres pourraient être dictés par le souci de ne pas donner prise à de nouvelles erreurs. S'il en est ainsi, antonino en toutes lettres a conduit à rejeter aug pio à la ligne suivante. Ce serait donc par accident qu'apparaîtrait cette disposition qui rappelle la famille sud aux lignes 4 et 5 , mais elle n'aurait pas été abandonnée, pour la dernière ligne, par un familier de la version sud. En fait, cette version, disposée en sept lignes, interponcluée et surlignée, est bien une adaptation de la version nord.

L'étude de ces trois milliaires nous permet de conclure : le texte "central" a été interprété deux fois par un familier de la version nord, une fois par un familier de la version sud.

L'existence de ces trois textes pouvait faire penser que la gravure des milliaires avait été confiée à trois ateliers, nord, centre et sud. Notre analyse nous oriente, on le comprend, vers deux ateliers, puisqu'il nous semble que le texte "central", qui n'est en fait 
qu'un texte correct, a été interprété deux fois avec les habitudes de nord et une fois avec celles de sud.

\section{LA FABRICATION DES INSCRIPTIONS}

On peut admettre que la série sud, qui commence au moins à MP XVII L 33 et allait jusqu'à MP XXXIII L 43, comptait ces 17 milliaires XVII-XXXIII au moins.

Au nord, il y avait 17 milliaires au nord d'Alba. Cette série comptait primitivement au moins les milliaires de IV à XVII (soit 14 milliaires), plus peutêtre les I-III au nord d'Alba (soit alors 17 milliaires), plus peut-être d'autres plus au nord, avec la possibilité de lacunes, si on admettait que des milliaires comme le N XII L25 soient, pour une raison qui nous échappe, à rattacher à la série "centre».

Cette répartition entre nord et sud laisse à première vue la place à une série "centre», avec le texte "central". Elle aurait pu comporter les milliaires I sud à XVI sud, plus quelques pierres éparses du type de N XII, et nous aurions donc trois groupes de milliaires sinon strictement égaux, du moins à peu près équilibrés : 17 ou plus pour sud, 14 ou plus pour nord, 16 environ pour centre, chacun traitant un texte particulier. L'homogénéité du travail de "sud" et de "nord" nous conduisant à parler d'atelier de gravure, on pourrait donc penser que la gravure des 50 (N I-XVII + S I-XXXIII) et quelques autres (N XVIII et suivants) a été répartie en trois lots à peu près égaux, entre trois ateliers.

Mais il y a une objection contre ce chiffre : c'est l'hétérogénéité des milliaires connus pour utiliser le texte "central». Ils n'ont guère en commun, en effet, que le texte. Leur version est entièrement différente : abréviations, interponctions et surlignages, dispositions, tout diffère.

En fait, nous avons remarqué que deux de ces milliaires nous rappellent les habitudes de l'atelier nord, tandis que l'autre évoque celles de l'atelier sud.

Enfin, nous avons à tenir compte du fait que les textes nord et sud sont incorrects, nord parce qu'il intervertit antonino et augusto, sud parce qu'il oublie caesar, et du fait qu'en revanche le texte "central" est le seul correct.

Nous pouvons donc émettre une hypothèse de travail : le lexte correct est une correction.

Il faudrait alors supposer qu'on ait averti les ateliers déjà en plein travail de ne pas oublier le mot caesar, pour l'atelier sud, et de ne pas intervertir antonino augusto, pour l'atelier nord.

En ce qui concerne caesar, l'hypothèse explique- rait qu'au sud, en L 32 , le mot semble introduit après coup, avec les conséquences que nous avons vues; mais elle expliquerait aussi que le mot ait été écrit, ce qui est exceptionnel, en toutes lettres: mieux valait désormais en faire un peu trop que pas assez.

En ce qui concerne l'interversion, au nord, la correction amenait à refaire la disposition.

Supposons maintenant que, dans chaque atelier, un personnage que nous appelons l'ordonnateur ait donné un modèle d'ordinatio aux graveurs. S'il s'apercevait ou s'il était averti d'une erreur, il refaisait un second modèle. Ce second modèle prenait en compte la correction, il était largement tributaire du premier modèle, mais il n'y avait pas de raison qu'il essaie de le reproduire autant que faire se pouvait. Cette hypothèse explique, nous semble-t-il, les caractères particuliers des milliaires qui ont le texte correct, soit N XII et S X pour la série nord, S XIIXIII pour la série sud.

Nous remarquons que la série "nord" dépasserait ainsi quelque peu, de 10 milles au moins en direction du sud, la ville d'Alba. Il n'y a pas là matière à réfuter notre hypothèse, mais au contraire à la consolider.

En effet, dans l'hypothèse des deux ateliers, ceux-ci auraient produit :

- nord : N XVIII et autres au nord; N XVII à I; S I à X ; peut-être S XI et S XII, soit 27, 29 milliaires ou plus, avec les milliaires au nord de N XVII;

- sud : S XII/XIII à S XXXIII, 21 ou 22 milliaires (peut-être 23 avec $\mathrm{S} \mathrm{XI}$ ).

Nous laissons de côté la question d'un milliaire 0 , qui aurait été, dans la ville d'Alba, le point de départ du compte des milles.

Si "nord" avait reçu les commandes des milliaires au nord d'Alba, et "sud" la commande des milliaires du sud, les deux commandes auraient été très déséquilibrées, 17 à 21 milliaires pour nord, 33 pour sud. Mais ce n'est pas le cas, et dans la mesure où nous rattachons $\mathrm{S} X \mathrm{~L}$ 31 à l'atelier nord, les deux groupes sont mieux équilibrés.

Pas strictement, dira-t-on. Mais regardons maintenant la carte (fig. 13). La route des Helviens comporte trois parties : un segment nord-sud suit le cours du Rhône; un segment est-ouest traverse Alba; un nouveau segment nord-sud pique droit vers Nìmes. Or la route vire vers le sud au milliaire S X, où se trouvait aussi le carrefour (voir annexe I, p. 76) de la voie romaine qui conduit vers Aubenas, la haute vallée de l'Ardèche, Pont-de-Labeaume, et les cols des Cévennes, une des grandes voies de 
communication des Helviens ${ }^{13}$. Il n'est pas malaisé d'imaginer que l'adjudication de la préparation des milliaires ait fixé comme limite ce point important entre tous. S'il en était bien ainsi,

- Nord devait préparer :

X I à XVII $=17$ milliaires,

$\mathrm{S}$ I à $\mathrm{X}=10$ milliaires,

soit 27 milliaires (plus quatre au plus au-delà de $\therefore$ XVII, comme nous essayons de le démontrer plus loin, annexe II, p. 78);

- Sud devait préparer :

$S$ XI à $\mathrm{S}$ XXXIII $=23$ milliaires.

\section{LES ÉTAPES DE LA FABRICATION}

Nous avons successivement parlé de "familles" de milliaires, puis plus précisément d'«ateliers». En effet, toutes les caractéristiques dont nous avons fait état concernent la mise en place de l'inscription sur la pierre, qui n'est pas proposée au niveau de la commande de la cité, car toutes les inscriptions seraient identiques, mais qui suppose un cadre concret où travaille le personnage qui s'en charge, et ce cadre concret peut ètre appelé "atelier".

On pourrait bien sûr admettre que la transformation du texte en version est l'œuvre du graveur des caractères. Mais, sous réserve de constatations ultérieures, l'homogénéité des familles n'est pas due au travail du graveur.

Il faut noter ici que nous ne connaissons que 18 milliaires et que sur ces 18 , nous ne pouvons raisonner qu'à partir de deux familles homogènes, l'une de 6 , l'autre de 8 milliaires. Mais actuellement, et sous réserve d'un examen plus approfondi de ces milliaires connus, nous n'identifions pas de "main" de graveur. C'est-à-dire que nous admettons provisoirement que plusieurs graveurs travaillaient, même dans le cadre d'une famille homogène. L'homogénéité de la famille ne vient donc pas des habitudes du graveur, mais de l'unicité de la version qui lui est donnée à graver.

Cette version ne lui est pas tracée sur la pierre : l'erreur de L 33 ANOTONINO pour ANTONINO ne s'explique que parce que le graveur grave lettre à lettre en regardant son modèle. Travaillant ainsi, il est évidemment égaré par la séquence IANANTO, il écrit IANANOTO et sa faute est irréversible.

Comment imaginons-nous son modèle? Deux supports de l'écriture sont peu coûteux et entre

13 Lacxerots, 1983, p. 119-122.

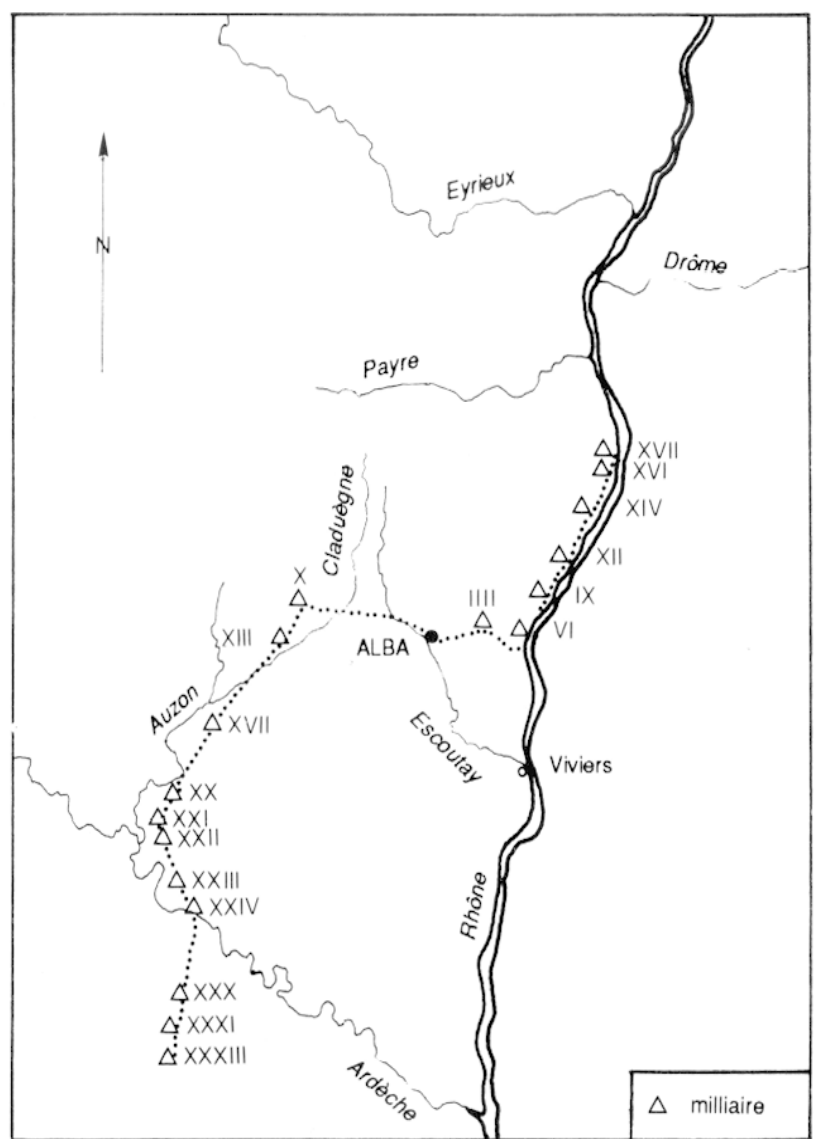

Fig. 13 - La voie des Ilelviens, croquis de situation (d'après A. Blanc).

toutes les mains : le tesson sur lequel on écrit à l'encre; la tablette, enduite ou non de cire, sur laquelle on grave. La tablette, qui suggère un champ épigraphique rectangulaire, est mieux adaptée à servir de modèle épigraphique que le tesson.

Nous imaginerons donc que l'atelier comporte un ordonnateur, qui prépare la version, et des graveurs qui la transcrivent sur la pierre. Dans ce cas, l'ordonnateur fournit évidemment un modèle unique, ce qui explique bien les ressemblances d'ensemble du résultat et les quelques variantes que le graveur se permet. Quand on avertit l'ordonnateur qu'il a fait une grosse erreur, il refait son travail en fonction de ses habitudes, mais sans être totalement lié par sa première série de modèles.

Il s'agit bien sûr d'une hypothèse de travail. Mais si elle se vérifiait, nous aurions fait une meilleure connaissance avec plusieurs personnages anonymes, dont la personnalité se dessine par quelques traits. 
Les autorités de la cité ayant pris la décision ${ }^{14}$ de borner la route, un des magistrats a été probablement plus particulièrement chargé du contrôle de l'opération. Peut-être est-ce lui qui a protesté contre les erreurs commises, à moins que ce ne soit un entrepreneur responsable du travail pour l'avoir pris en adjudication. En tout cas, ce qui est fait est fait, on ne recommence ni n'abandonne les milliaires "fautifs".

Les carriers livrent des pièces finies. On n'exige pas d'eux qu'elles soient de dimensions strictement identiques. Néanmoins, ils sont capables de fournir un lot homogène de cinquante à cinquante-quatre supports.

Il y a deux ateliers de gravure. Chacun a un ordonnateur, peut-être simplement le patron, qui reçoit le texte. S'il est écrit, s'il comporte peut-être déjà quelques abréviations très usuelles, imp, aug, $p p, \cos , m p$, qui se retrouvent dans tous nos milliaires, ce texte n'est pas disposé. Peut-être est-il lu et pris sous la dictée. D'après ce texte, dans chaque atelier, l'ordonnateur prépare une ordinatio. Il utilise certainement un champ rectangulaire ou approximativement rectangulaire, probablement une tabella en bois et cire, peut-être un ostracon écrit à l'encre. S'il y a plusieurs graveurs, ce modèle est peut-être préparé en plusieurs exemplaires. Ces ordonnateurs ne sont pas de fins connaisseurs des titulatures impériales. Nous ne voyons pas en eux des spécialistes de l'épigraphie, plutôt d'honnêtes artisans, les patrons de l'atelier où l'on grave.

Les graveurs disposent d'un bon savoir-faire pour ce qui concerne les lettres elles-mêmes. Ils copient leur modèle lettre à lettre (anatonino). Leur latitude est limitée : ils peuvent cependant remplacer un iiii par iv, couper des lettres finales (aelio|ael; antonin|anton; hadrian|hadr), décaler légèrement la disposition (S XX L 34 anto|nino au lieu de an|tonino du reste de la série, décalage qui se répercute ensuite). Dans l'ensemble, ils font un travail consciencieux et même élégant.

Nous ne savons rien d'un dernier et indispen-

14 Il n'est pas de notre propos de nous interroger sur le processus de décision lui-mème. Si nous remarquons que dans une courte période (TR P VII COS III, mais III ne repose plus aujourd'hui que sur les lectures de Hirschfeld en 1983 et 1985 ; TR P VII cos IIII; TR P VIII COS IIII), on érige d'autres milliaires en Narbonnaise (KöNıG, 1970, p. 79-82) on peut penser que la cité répondait en fait à un vou d'une autorité supérieure (qui n'explique pas la pose de tous les milliaires du règne, en particulier de la série TR $\mathrm{P}$ II COS II). Ce vœu date de l'année 144. Nous disons un vou et non un ordre, puisque d'autres cités n'ont pas imité les Ilelviens. sable artisan, le "rubricateur» : c'est lui qui peint les lettres, ce qui va permettre aux voyageurs d'utiliser les milliaires. Les voyages auraient été vraiment lents dans l'Antiquité, si les voyageurs avaient dû passer, devant chaque milliaire, autant de temps que nous pour être renseignés!

La cité d'Alba décidant de poser des milliaires :

- dispose du programme de pose, qui permet de savoir qu'il faudra 50 (à 54) milliaires;

- agit directement ou sous-traite, ce que nous ignorons.

En tout cas, une commande homogène de la totalité des blocs est passée à une carrière.

A partir des blocs parallélépipédiques, il va falloir tailler les supports. Imaginerons-nous que cela se faisait dans la carrière ou dans les ateliers de gravure? Nous répondons : dans la carrière, parce que tous les supports sont, autant que nous sachions, comparables (les différences de dimensions venant partiellement des différences de dimensions des blocs eux-mêmes), et que nous n'avons en particulier pas noté de différences entre des supports du nord et des supports du sud; parce qu'aussi, ce travail de taille est bien dans les cordes des carriers, et parce que nous constatons qu'ailleurs, ils livraient les supports mouluration comprise ${ }^{15}$, quand on la leur avait commandée.

Ces supports sont répartis en deux lots à peu près égaux, entre deux ateliers de gravure, que nous appcllcrons "nord" et "sud"; nord a la charge de tous les milliaires à placer au nord d'Alba, et les premiers milliaires à placer au sud de la ville, jusqu'au carrefour où se détache vers l'ouest la voie de la montagne, où sera placé le $\mathrm{S} X$.

L'ordonnateur de nord distribue aux graveurs une version où il a interverti antonino et augusto; l'ordonnateur de sud oublie caes.

Plusieurs milliaires sont déjà terminés quand les erreurs sont découvertes. Ce serait une étrange coïncidence que chacun des deux ordonnateurs se fût aperçu à peu près simultanément d'une erreur de sa version. C'est donc le commanditaire de l'opération qui a procédé à un contrôle. L'ordonnateur de chaque atelier modifie sa version, et le travail est achevé sur ces nouvelles données.

Tout est peut-être ${ }^{16}$ terminé avant qu'Antonin soit trib pot viii. On a, dans ce cas, fait vite ${ }^{17}$.

15 KöNıG, 1970, ns 183, 212, 227.

16 Car si on admet la thèse de la correction en cours de travail, on aurait peut-être mis à jour le nombre des puissances 


\section{COMPARAISONS}

Nous devons maintenant vérifier que la théorie que nous avons esquissée s'applique à d'autres séries que celle de la cité des Helviens.

Nous admettons :

- 1 qu'une autorité responsable prescrivait qu'un texte donné serait utilisé ;

- 2 qu'un ordonnateur était responsable de la version à inscrire sur les pierres; cette version comporte le choix des abréviations (sauf éventuellement des plus courantes, qui pouvaient déjà être données par le texte), de la disposition en lignes, décide de l'usage des interponctions et des surlignages, et éventuellement des ligatures; que si la commande était divisée, nous pouvons assister au travail de deux ou plusieurs ordonnateurs; que cette division du travail se reconnaît précisément à ses effets ;

- 3 que le ou les graveurs copiaient un modèle, avec peu d'autonomie: minimes modifications de disposition, modification de quelques abréviations, choix entre le chiffre IIII et le IV (et donc par analogie entre VIIII et IX, par exemple), utilisation des ligatures en fonction de la place disponible; il peut arriver aussi qu'un graveur modifie un alinéa soit en revenant trop tôt à la ligne par inadvertance ou parce qu'il a trop écarté, soit en voulant garnir une ligne où il a trop serré, mais on peut attendre que ces manifestations d'inattention, de maladresse ou d'embarras soient plus fréquentes quand le champ épigraphique est limité par une mouluration ${ }^{18}$.

tribuniciennes. Ce n'est pas certain, car on pouvait vouloir respecter le texte initial. Mais ce ne sont alors que les quelques milliaires corrigés qui ont pu déborder sur la VIII ${ }^{\mathrm{e}}$ puissance tribunicienne.

17 Le texte n'a pu être fixé qu'après qu'Antonin ait été désigné pour le $4^{\mathrm{e}}$ consulat qu'il allait prendre au $1^{\mathrm{er}}$ janvier 145. Il a été fixé suffisamment tôt pour que l'autorité responsable puisse penser que toute l'opération serait terminée dans le cours de sa $7^{\text {e }}$ puissance tribunicienne. Suffisamment tard aussi pour qu'elle pense qu'il était normal de dater les milliaires du $4^{\mathrm{e}}$ consulat. Faut-il dater la décision de la fin de l'année 144, avec peut-être un début du travail? et la pose effective des milliaires au tout début de l'année 145 , avec un règlement des dépenses sur l'année budgétaire 145 ?

Nous disons ici "tout début" sur la suggestion de G. di Vita, qui nous confirme dans l'idée que ce travail a été fait "vite". En effet, c'est probablement le 25 février 145 (ou très peu après, si on tient compte de la confirmation officielle) qu'Antonin prend sa 8 e puissance tribunicienne. Toute l'opération de pose des milliaires s'inscrit donc dans un délai de quelques mois, peut-être moins de six, ce qui ne nous semble pas invraisemblable.

18 Voir infra, p. 66, des cas où le graveur franchit la
En ce qui concerne les fautes et erreurs :

- 1 l'autorité responsable peut commettre une erreur administrative, sur le libellé d'une titulature par exemple;

- 2 les fautes de l'ordonnateur se situent plutôt au niveau des mots, oublis, interversions;

- 3 les fautes du graveur se situent plutôt au niveau des lettres, oublis, duplications, et des signes, interponctions ou surlignages oubliés ou parasites.

La vérification de cette théorie suppose l'utilisation de publications précises et fiables. Les épigraphistes s'en sont souvent tenus au texte, qui à lui seul est pratiquement inutilisable pour nous; leurs indications sur la version et sur la gravure sont souvent incomplètes, souvent réduites aux "fautes" concernant les mots et les lettres, mais les indications sur les autres particularités sont souvent négligentes, en particulier en ce qui concerne les interponctions (dont le relevé nécessite souvent un examen attentif de la pierre, et un grand soin dans la copie).

Néanmoins, nous pouvons utiliser quelques séries (en prenant dans ce qui suit la numération des milliaires de I. König).

\section{VOIE DOMITIENNE, AUGUSTE, L'AN 3 AVANT NOTRE ÈRE}

Tous les milliaires ont le même texte. Mais König note que les milliaires situés à l'est du Vidourle ont une version (nous employons notre propre vocabulaire) de six lignes, tandis que ceux de l'ouest ont cinq lignes.

\begin{tabular}{|l|l|}
\hline \multicolumn{1}{|c|}{ Beaucaire au Vidourle } & \multicolumn{1}{|c|}{ Vidourle à Montpellier } \\
\hline $\begin{array}{l}\text { imp caesar } \\
\text { diui f aug pontif } \\
\begin{array}{l}\text { cos designat xiii } \\
\text { imp xiiii tribunicia } \\
\text { potestate xx }\end{array}\end{array}$ & $\begin{array}{l}\text { imp caesar } \\
\text { diui f aug pont } \\
\text { max cos xii cos } \\
\text { design xiii imp xiiii } \\
\text { trib pot xx }\end{array}$ \\
$\begin{array}{l}n^{\text {os }} 172,179,186,190,200, \\
211,214,218,223\end{array}$ & $n^{08} 224,231,236,240$ \\
\hline
\end{tabular}

mouluration. Nous avons signalé, aux portes du camp de Bu Njem-Gholaia, un cas où un graveur perce une moulure et transforme un champ rectangulaire en tabella ansata, pour inscrire dans cet élargissement du cadre les lettres qu'il n'a pas voulu reporter à la ligne suivante (R. Rebuffat, Les inscriptions des portes du camp de Bu Njem, Notes et Documents IV, Libya Antiqua, IX-X, 1972-1973, p. 111-114). 
Les abréviations et la disposition sont constantes. En ce qui concerne les interponctions, nous ne savons pas si les transcriptions sont fiables. Dans la série est, elles semblent sporadiques (deux en 186 ; trois en 211 ; une en 218). Dans la série ouest, elles semblent beaucoup plus fréquentes : trois en 224 ; quatre en 240 ; douze en 230 ; cinq? en 233). Les surlignages ne sont d'aucun secours, car nous nous demandons s'ils ont été notés avec soin.

Les deux milliaires ouest 230 et 233 offrent une variante à la ligne 5 , potest au lieu de pot: deux hypothèses, initiative de graveur, ou variante au niveau de la distribution aux graveurs des exemplaires de la version?

Une nouvelle étude de ces milliaires est nécessaire ${ }^{19}$. En attendant, nous pensons que deux ateliers ont été au travail, chacun dépendant pour sa version de son propre ordonnateur. Chacun était responsable d'une section de la route topographiquement déterminée, la limite étant au Vidourle. Nous avons affaire ici au cas qui se rapproche le plus nettement de celui des milliaires helviens. Il montre que la pratique d'une division du travail ainsi conçue pourra être indentifiée dans d'autres cas. II ne s'ensuit pas, bien sûr, qu'on faisait toujours ainsi.

\section{VOIE DOMITIENNE, TIBÈRE, 31-32}

Tous les milliaires connus ont le mème texte, sept numérotés à partir de Nîmes $\left(\mathrm{n}^{\mathrm{os}} 171,173,180\right.$, $181,184,189,191)$, neuf numérotés à partir de Narbonne ( ${ }^{\text {os }} 210,229,232,234,238,239,241,242$, 247). Tous sauf trois ont la même version. Un seul ordonnateur est donc intervenu ${ }^{20}$.

19 D'autres milliaires sont connus en Narbonnaise pour la même date. Le 114 (numéros de König) de la voie d'Agrippa, le 26 de la "Via Aurelia * et le 72 de la voie de Forum Voconii à Riez donnent le même texte. Deux ont des versions complètement différentes de celles de la Via Domitia. En revanche, le 26 de la "Via Aurelia" a la mème version que la Via Domitia section est. Cependant, cette version n'est assurée que par deux lignes, et ce milliaire porte une indication de distance, ce qui n'est jamais le cas pour ceux que nous avons cités plus haut. Il est donc prudent de ne rien conclure de cette analogie.

20 On se demande si les chiffres de distance n'ont pas été ajoutés après coup. Mème si ce n'est pas le cas, voici une occasion où l'étude de la main permettrait de savoir si un seul ou plusieurs graveurs étaient à l'œuvre. Faut-il également penser que le chiffre ètait gravé quand le milliaire était in situ? Et (nous nous adressons ici aux graveurs sur pierre) ne pourrait-on, dans ce cas, savoir si on gravait sur le milliaire couché ou debout? On peut donc en tout cas remarquer que le chiffre de distance est omis sur un milliaire, le 171. Il est peu probable qu'un graveur ait sauté ou omis un milliaire : faut-il penser à une erreur de répartition du travail, qui prouverait bien qu'il n'y avait pas qu'un seul graveur?
Trois milliaires du même texte ont des versions toutes trois différentes:

\begin{tabular}{|c|c|c|}
\hline $\begin{array}{l}\text { ti caesar } \\
\text { diui augusti f } \\
\text { angustus } \\
\text { pontifex } \\
\text { maxumus } \\
\text { tribunicia } \\
\text { potestate xxxiii } \\
\text { refecit et } \\
\text { restituit }\end{array}$ & $\begin{array}{l}\text { tib caes } \\
\text { diui aug } \\
f \text { aug pont } \\
\text { maximus } \\
\text { trib pot xxxiii } \\
\text { refecit et } \\
\text { restituit }\end{array}$ & $\begin{array}{l}\text { tib caesar } \\
\text { diui augusti } \mathrm{f} \\
\text { augustus } \\
\text { pontifex } \\
\text { maxumus } \\
\text { tribunicia } \\
\text { potestate } \\
\text { xxxiii } \\
\text { refecit }\end{array}$ \\
\hline 55 & 166 & 248 \\
\hline $\begin{array}{l}\text { Saint-Cannat } \\
\text { entre Aix } \\
\text { et Tarascon }\end{array}$ & $\begin{array}{l}\text { Trinquetaille } \\
\text { entre Arles } \\
\text { et Nimes }\end{array}$ & $\begin{array}{l}\text { Les Castans } \\
29 \mathrm{MP} \\
\text { Narbonne }\end{array}$ \\
\hline
\end{tabular}

De pareilles différences ne relèvent pas des graveurs. En fait, le $5 \overline{5}$ est à l'est du Rhône (König l'attribue à la Via Aurelia, et non à la voie domitienne); 166 est à l'est de Nîmes, et il n'entre donc pas non plus dans la série cohérente citée plus haut. On peut supposer que ces deux milliaires n'ont pas été gravés dans l'atelier responsable des milliaires 171-247, et de part et d'autre du Rhône, on a certainement recouru à deux ateliers différents.

Pour le 248, il se situe au-delà vers l'ouest du dernier milliaire cité plus haut. Faut-il invoquer une raison comparable à la précédente ou penser qu'il s'agit d'un rajout ultérieur au programme initial? Il vaut mieux dans ce cas ne pas conclure.

Notons que la variation maximus/maxumus ne se situe pas au niveau du texte, mais de la version ou peut-être du graveur.

\section{VOIE DOMITIENNE, CLAUDE, 41}

Ces magnifiques milliaires, tous de même texte, ont le grand intérêt pour nous de présenter un cadre mouluré pour le champ épigraphique. Ceci nous permet de vérifier que le milliaire était livré de carrière avec son cadre, car le graveur a souvent été gêné par lui :

183: le graveur lie maladroitement les deux dernières lettres de claudius;

212 : la dernière lettre de claudius, de caesar et de trib sont à l'extérieur du cadre;

227 : le b de trib chevauche le cadre.

Nous pouvons donc considérer que les deux groupes qui ont même texte et même version, à l'exception de la fin du mot germanicus, dépendent en fail d'une seule version, le graveur pouvant employer soit germanicus, soit germanic selon la 
nécessité. Au surplus. la répartition des deux formes montre quil n y a pas lieu de les classer en deux séries.

\begin{tabular}{|l|l|l|}
\hline & $\begin{array}{l}\text { ti claudius } \\
\text { drusi f caesar } \\
\text { aug germanicus } \\
\text { pontif max trib } \\
\text { pot cos desig ii } \\
\text { imp ii refecit }\end{array}$ & germanic \\
\hline Beaucaire-Nìmes & $169,182,192$, & $170,183,187$, \\
& 195,201 & 196,202 \\
\hline Nìmes-Narbonne & 226,235 & $\begin{array}{l}208,215,216, \\
217,227,228\end{array}$ \\
\hline
\end{tabular}

On ne peut pas classer le 194, mutilé ou mal lu. ni tenir compte du 167 d'Arles, apparemment de mème texte et de mème version (sauf pont? pour pontif), mais perdu.

Le cadre est responsable d'autres non-respects de disposition, dus au graveur. En 212, desig arrivant trop près du cadre, le graveur est contraint d'écrire desig | ii imp, serrant d'autant la dernière ligne de l'inscription.

Nous pouvons aussi détecter une erreur d'inattention. Le graveur, en 243, a écrit potestate (hapax dans la série) en toutes lettres, ce qui fait qu'il a dû rejeter desig à la ligne suivante :

$$
\begin{aligned}
& \text { polestate [cos] } \\
& \text { desig ii imp ii r [efec] }
\end{aligned}
$$

Il s'agit d'une distraction, qui nous indique d'autre part que ce graveur connaissait parfaitement le sens des abréviations (et qu'il interprétait pot comme devant être développé à l'ablatif).

Enfin, nous pouvons interpréter comme une maladresse du graveur la disposition de 176-177 Jonquières :

\section{pontif max trib pol cos desig ii imp ii refecil}

Ayant trop serré la ligne 4, puis la ligne 5 , il se trouve avec une ligne 6 déséquilibrée.

Dans l'ensemble, les milliaires de Claude permettent de faire l'hypothèse qu'une version unique a été proposée. Seule l'étude archéologique des pierres pourrait démontrer qu'elles ne viennent pas d'un seul atelier.

\section{VOIE DOMITIENNE, ANTONIN, EN 145}

Un a de nouveau affaire à des milliaires qui offrent un cadre mouluré.

On ne connaît que treize milliaires de cette série.
Mais le 174 est attribué à la série d'après la dimension du support; le 175 , le 188 et le 193 sont restitués d'après le 185 : le 203 est perdu et c'est simplement une attribution vraisemblable : le 213 est perdu: le 220 est restitué d'après le 207 . On ne peut donc raisonner que sur les 185. 199 (perdu, mais lu), 206, 207, 209 et 219 (qui ne conserve quelque chose que des trois premières lignes), et faire plutôt une demande d'enquête qu'une hypothèse.

Cependant, on note que le 185 , entre Beaucaire et Nìmes, adopte une autre version que le 206, entre Nìmes et Narbonne.

\begin{tabular}{|l|l|l|}
\hline 185 & & 206 \\
\hline imp caesar & 1 & imp caesar \\
diui hadrian f & 2 & diui hadriani \\
t aelius hadrian & 3 & $\mathrm{f}$ t aelius hadri \\
antonin aug pius & 4 & anus antoninus \\
pont max trib potes & 5 & aug pius \\
viii imp ii cos iiii & 6 & pont max trib pot \\
pp restituit & 7 & viii imp ii cos iiii \\
& 8 & pp restituit \\
\hline
\end{tabular}

Le 185 étant de plus interponctué, tandis que le 206 ne l'est pas.

$\mathrm{Au} 185$ peuvent se rattacher:

- le 199 perdu, où les lignes 5-7 pont max trib pol viii imp ii cos iiii $p p$ restituit

semblent une simple variante de gravure par rapport à 185 (sans qu'on sache lequel des deux est le plus fidèle à l'ordinatio);

- le 207 qui a, par rapport au 185, deux variantes minimes (antoninus et trib pot), et une ligne de plus : $p p \mid$ restituit, ce qui s'explique parce que le graveur, ayant trop serré les lignes, a trop de blanc vers le bas du cadre, et crée deux lignes au lieu d'une ;

- le 219, dont la seconde ligne diui hadrianif, avec un $f$ tracé sur la moulure par le graveur, qui ne s'est pas cru autorisé à le reporter à la ligne, indique bien que le modèle suivi était ainsi.

Au 206 peut se rattacher le 209, avec deux variantes minimes, antonin pour antoninus et pot rejeté à la ligne suivante, sans autre modification de la disposition, et au contraire avec aug pius occupant une ligne. Ce rattachement est d'autant plus justifié que le surlignage irrationnel VIII II IIII de 206 se retrouve en 209 (et non en 207 , où on a VIII II IIII, ce dernier chiffre étant évidemment à vérifier).

A ce point, on peut faire l'hypothèse de deux ordonnateurs. Mais contrairement à ce que nous 
avons admis jusqu'ici, les deux ateliers n'ont pas distribué leurs milliaires sur deux sections de routes différentes, mais semblent les avoir entrecroisés au hasard. En revanche, on peut se demander si les chiffres des MP, qui semblent tous, pour autant qu'on le voie, de la même main, n'ont pas été tracés après l'installation des milliaires.

\section{OBSERVATIONS MÉTHODOLOGIQUES}

En présentant les leçons que nous pouvons tirer de notre étude, nous souhaitons signaler les informations qui nous ont manqué ou les habitudes, prises depuis longtemps dans la transcription des inscriptions, qui nous ont gêné.

\section{ÉTUDE ARCHÉOLOGIQUE}

Il nous a manqué les indications suivantes.

- Des mesures (nous avons trouvé que le plus simple est de se munir d'un croquis préalable, et de le renseigner sur place).

- Des indications sur la base du milliaire, et, secondairement, sur le procédé employé pour passer éventuellement d'une base quadrangulaire à un fût arrondi.

- Des indications précises sur le fût des milliaires. Il est vrai qu'il est très difficile à mesurer, car il est rare que sa section soit un cercle : c'est en général plutôt un ovale; d'autre part, le fût est souvent légèrement tronconique. On peut prendre deux diamètres, l'un perpendiculaire à l'axe de lecture de l'inscription, l'autre parallèle et se munir d'autre part d'un mètre souple de couturière pour prendre les trois circonférences, en haut, au milieu et en bas (et d'une calculette pour diviser par 3,14159 et obtenir les diamètres correspondants, qu'il vaut mieux calculer sur place, afin de les comparer tout de suite à la mesure directe des diamètres supérieurs).

- Des indications, champ par champ, sur la nature du travail : épannelage, polissage. Si on pouvait déterminer les outils utilisés, ce serait encore mieux!

- Des notes sur la façon dont la pierre accepte le travail de gravure : facile à polir, alvéolaire, coquillière, par exemple.

\section{ÉTUDE ÉPIGRAPHIQUE}

\section{Milliaires isolés}

- Nous nous sommes rendus compte de l'importance des interponctions, qui dans d'anciennes copies sont souvent des ponctuations ou des ornements dus à l'initiative des lecteurs. Dans des copies plus récentes, il arrive que ne soient pas notées les lacunes de la pierre qui permettent de penser qu'une interponction a pu disparaître, ainsi que le caractère éventuellement alvéolaire de cette pierre, qui peut produire des erreurs.

- Les surlignages, mais aussi leurs anomalies, en particulier leur longueur par rapport aux caractères qu'ils devraient couvrir, ont la même importance que les interponctions.

- Il est essentiel de savoir si le chiffre des distances, quand il figure sur la pierre, est de la même main que le reste de l'inscription, de façon à savoir si sa gravure constitue une étape indépendante dans le processus épigraphique.

\section{Séries}

- Dans d'anciennes publications, on trouve souvent l'inutilisable mention "même texte». En fait, il faut pouvoir disposer d'une description précise de chaque milliaire.

\section{TRANSCRIPTION}

- Nous avons constaté que les transcriptions en capitales sont pratiquement inutilisables. Ne séparant pas les lettres, elles sont peu lisibles, et l'expérience prouve qu'on ne les consulte que dans les cas où on doit raisonner sur la lecture elle-même matériellement définie : et dans ce cas, il est suffisant de se borner, dans le commentaire, à la transcription du passage litigieux. Lorqu'elles sont encombrées de signes diacritiques, ces signes gênent sinon la lecture, du moins les comparaisons. Peu importe, on peut les conserver si on y tient : mais le raisonnement s'appuie surtout sur la lecture de l'inscription.

- Pour cette lecture, les habituels compléments entre parenthèses sont encombrants, et particulièrement gênants. Si à l'extrême limite un problème se pose, il a sa place dans le commentaire. Une lecture encombrée de parenthèses devient paradoxalement illisible; trop longue, elle ne peut plus être disposée en respectant les alinéas, c'est-à-dire la disposition même du texte. Or nous avons vu qu'il est absolument nécessaire de prendre visuellement conscience de cette disposition, ce que ne permet pas la lecture développée.

Une lecture courte peut être à l'occasion disposée sans alinéas, avec les classiques barres verticales de séparation, quand cela n'aura pas d'inconvénient pour le raisonnement.

- Comme on a vu l'importance des interponctions et des surlignages, il y a intérêt à les porter sur la lecture.

Dans la transcription des lectures, nous avons trouvé avantage à séparer les mots par un blanc (ou 
par l'interponction). Le seul inconvénient est que cela ne permet pas de comparer directement le nombre de lettres d'une ligne, mais on sait que ce nombre de lettres est de toute façon trompeur quand il s'agit d'apprécier une longueur. Le gros avantage est qu'on peut raisonner sur une lecture facile.

- Les majuscules gênent les photographies des lectures auxquelles l'œil procède quand il veut les comparer. D'autre part, quand il s'agit d'apprécier les réactions d'un ordonnateur vis-à-vis du texte qu'on lui a transmis, et d'un graveur vis-à-vis du travail de l'ordonnateur, il faut bien se dire que ni l'un ni l'autre ne se trouvaient devant des majuscules et qu'ils n'avaient pas la moindre conscience qu'un tel code pût exister. Si nous écrivons un texte doté de majuscules, et une ordinatio dotée de majuscules, nous créons un document très éloigné de celui qui pouvait provoquer les réactions de l'ordonnateur et du graveur.

- La ponctuation moderne a le même inconvénient. Un texte ponctué aurait conduit un ordonnateur à réagir de façon toute différente dans son travail, et il en serait de même pour une ordinatio ponctuée.

En un mot, nous aurions souhaité trouver pour chaque milliaire un document lisible, ayant tous les avantages d'une lecture en minuscules, et délivré des majuscules, de la ponctuation moderne, et des développements d'abréviations; un document offrant à la comparaison un texte court et facile à lire, pour lequel la matérialisation essentielle des alinéas soit aisée, et qui conserve des transcriptions en capitales habituelles les avantages précieux que sont la notation des interponctions et des surlignages.

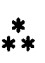

Un exemple nous permettra de faire mieux comprendre ce que nous avons essayé d'expliquer.

Voici la lecture de $R$. Lauxerois pour les milliaires 21 et 26 :

Imp(eratore) Caes(are) T(ito) / Ael(io) Hadr (iano) / Aug(usto) Antonin(o) / Pio P(atre) P(atriae) trib(unicia / pot(estate) VII / c/o(n)s(ule) IV] | M(illia) p(assuum) XVI.

Imp(eratore) Caes(are) / T(ito) Aelio Hadr (iano) / Aug(usto) Antonin(o) / Pio P(atre) P(atriae) | Trib(unicia pol(estate) VII / co(ns)s(ule) [I] III | M(illia) p(assuum) VIIII.

Si nous les transcrivons avec notre système de présentation, cela donne: imp caes $\mathrm{t}$ | ael hadr | aug antonin | pio $p \mathrm{p}$ trib | pot vii | $c$ cos] iv | $m p$ xvi

imp caes | t aelio hadr | aug antonin | pio $p p \mid$ trib pot vii $\cos [$ [i]iii $\mid m p$ viiii

Le système traditionnel entraîne des inconvénients (Trib/trib; consule/conssule; le choix forcé d'un cas pour tribunicia). Ils sont inéluctables, mais secondaires. L'essentiel est que la comparaison est difficile. En revanche, notre transcription permet de voir d'un seul coup d'œil une différence : la présence en 21 de $t$ (ito) à la première ligne. Cette anomalie invite aussitôt à vérifier toute la série, où $t$ (ito) est toujours à la seconde ligne. L'anomalie devient alors un hapax qui invite à revoir la pierre. On s'aperçoit alors que le déchiffrement est fautif, et qu'en 21 t(ito) est en fait à la seconde ligne.

La lecture développée a un autre inconvénient : la restitution antonin(o) estompe une différence essentielle entre cette famille nord, où on trouve toujours anton ou antonin (sauf pour le milliaire corrigé 31 ) et la famille sud, où anlonino est toujours en toutes lettres.

Si nous portons les interponctions, la différence entre les deux familles nord et sud, comme on le voit par un court exemple, apparaît très clairement :

26 : imp.caes | t.aelio. hadr | aug.antonin | etc.

35 : imp tito | aelio ha | drian an | tonino | etc.

Il en est évidemment de même quand on retranscrit les surlignages.

C'est bien sûr la présentation respectant les alinéas et l'intégralité de la version proposée au graveur qui rend les meilleurs services:

\begin{tabular}{|c|c|c|c|}
\hline nord 21 & nord 21 & nord 26 & sud 35 \\
\hline $\begin{array}{l}\text { nouvelle } \\
\text { lecture }\end{array}$ & $\begin{array}{l}\text { ancienne } \\
\text { lecture }\end{array}$ & & \\
\hline $\begin{array}{l}\operatorname{imp} \text { caes } \\
\text { [t]ael. hadr } \\
\text { aug.antonin } \\
\text { pio.p.p.trib } \\
\text { pot vii } \\
\text { c[os] iv } \\
\text { m.p xvi }\end{array}$ & $\begin{array}{l}\text { imp caes t } \\
\text { ael.hadr } \\
\text { aug. antonin } \\
\text { pio.p.p.trib } \\
\text { pot vii } \\
\text { c[os] iv } \\
\text { m.p xvi }\end{array}$ & $\begin{array}{l}\text { imp.caes } \\
\text { t.aelio. hadr } \\
\text { aug. antonin } \\
\text { pio.p.p } \\
\text { trib.pot.vii } \\
\text { cos. [i]iii } \\
\text { m.p.viiii }\end{array}$ & $\begin{array}{l}\text { imp tito } \\
\text { aelio ha } \\
\text { drian an } \\
\text { tonino } \\
\text { aug pio } \\
\text { p p trib pot } \\
\text { vii cos iiii } \\
m \text { p xxi }\end{array}$ \\
\hline
\end{tabular}

L'anomalie de nord 21, par rapport à 26, saute alors aux yeux. D'autre part, la différence entre les deux familles nord et sud est évidente.

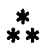

A vrai dire, la question est plus grave que la 
commodité de qui veut comparer deux ou plusieurs milliaires. $\mathrm{Si}$, pour juger d'une inscription, on en donne des présentations dont ni un graveur, ni un ordonnateur, ni un chef d'entreprise, ni un magistrat, ni un empereur n'auraient jamais pu avoir la moindre idée, on les trahit.

\section{OBSERVATIONS ÉPIGRAPHIQUES}

Dans ce qui suit, nord et sud désignent les deux familles que nous avons distinguées. Mais les sigles, $\mathrm{N}$ XVII et $\mathrm{S} X$, désignent les milliaires du nord et du sud d'Alba, puisque les milliaires sont numérotés à partir de cette ville.

\section{FAMILLE NORD}

\section{N XVII L 20 Cruas}

imp.caes | t.aelio. hadr | aug.anton | pio.p.p | trib. pot vii | cos iiii | m.p. xvii

Nous lisons anton et non antoni (Lauxerois: antoni). On peut croire voir une haste finale, mais ce n'est pas une lettre. D'autre part, rien n'incite à voir une ligature NI. Tous les autres milliaires ont en fait antonino, antonin, ou anton, tandis qu'antoni serait un hapax dans la série. anton est d'autre part attesté par N IV et XIV.

Le surlignage couvre bien les trois chiffres de vii (Lauxerois : il couvre les deux derniers chiffres).

\section{N XVI L 21 Cruas}

imp caes | [t]ael.hadr | aug. antonin | pio.p.p.trib | pot $\diamond$ vii | co[s] $\diamond i v \mid$ m.p $\diamond x v i$

Nous notons les $\diamond$ lacunes qui ont pu contenir une interponction (Lauxerois : imp caes $l \mid$ ael. hadr). antonin, inconnu de la série sud, est bien attesté au nord (N XII IX VI).

\section{N XIV L 22 Cruas}

Lecture traditionnelle :

imp caes | t ael hadr / aug anton | pio pp trib | pot vii | cos iv | $m$ [p xi]v
Les abréviations, la disposition et le surlignage intègrent complètement ce milliaire dans la série nord. L'absence des interponctions sur les lectures qui nous ont été transmises était donc particulièrement surprenante. La vérification n'a pas été facile : le milliaire était, pendant nos visites, au fond d'une baraque de chantier, dont nous n'avons d'abord pas trouvé la clé, que nous a finalement aimablement procurée M. le curé de Cruas. La situation du milliaire, au milieu de divers blocs, ne facilite pas son examen. Tout cela dit, nous avons pu lire :

imp $\diamond$ caes $\mid$ t.ael $\diamond$ hadr $\mid$ aug.anto $\mid$ pio $\diamond p p \diamond \ldots-. \mid$ le reste trop dégradé.

Il y a donc deux interponctions bien visibles, et quatre possibilitès $\diamond$ d'interponctions (dont une très probable après ael) dans des lacunes de la pierre, qui ont évidemment fait hésiter les commentateurs à lire des points. Le milliaire entre donc bien sans objections possibles dans la série nord.

\section{N XII L 25 Meysse}

...c -.. | [t.] ael. hadrian | antonin.aug | pio.p.p.trib po | test. vii.cos iiii | m.p.xii

Ligne 1 : le bas du C est visible. Impossible de savoir si on avait caes ou caesare. La place disponible suffirait pour caesare.

Ligne 3 : antonin.aug avec interponction.

Ligne 5: test.vii.cos iiii avec deux interponctions.

\section{$N X L 26$ Rochemaure}

\section{Ligne $3: \mid$ aug. antonin |}

On croit voir un tout petit 0 à la fin de la ligne, mais c'est une illusion. Mème toute petite, la lettre serait gravée avec les mèmes coups de burin francs que le reste du texte.

Ligne 6 : lire | cos.iiii | avec une interponction.

\section{N IV L 30 Route du Teil}

\section{Ligne 1 : imp.caes}

Le point après imp est presque effacé. Il n'y a rien après caes et d'ailleurs les milliaires considérés n'ont pas, sauf un, de point en fin de ligne. 


\section{$S X L 31$ Saint-Germain-en-Laye}

Rappelons qu'il s'agit, malgré sa position au sud d'Alba, d'un milliaire de la famille nord.

imp.caesare | t.aelio.hadr. | antonino | aug.pio | p p. trib pot vii $\cos \mid$ iiii.m.p $x$

Point après hadr, exceptionnel en fin de ligne.

Pas de point après m.p

Interponction douteuse après trib

\section{FAMILLE SUD}

\section{S XII | XIII L 32 Saint-Germain-d'Ardèche}

imp caesar | tito ael ha | dr antoni | no aug pio | pp trib pot | vii cos iii ! mp [?]

A la ligne 1, nous lisons comme König caesar. A la ligne 2, nous avons pu distinguer -.. ELHA. Cette lecture est très satisfaisante, car la coupe $h a \mid d r$ est caractéristique de la série sud.

A partir de la ligne 3 , nous avons pu vérifier que toutes les anciennes lectures sont exactes. Il ne s'agit donc pas d'une lecture influencée par celle des autres milliaires, comme le craint R. Lauxerois.

\section{$S X X L 34$ Pradons}

imp tito | aelio ha dr anto | nino [aug] | pio pp trib | pot vii ! $\cos$ iiii $\mid m p x x$

La lecture de König est exacte. Il est désormais impossible de lire les lignes 7 et 8 , et on ne distingue plus que le haut de la ligne 6.

\section{$S X X X$ L 41 Salavas}

[ligne 1] | [ligne 2] | [ligne 3] | [to]nin[o] | aug pio | pp trib pot : vii $\cos$ iv $\mid \mathrm{mp} x \mathrm{xx}$

Ligne 4 , il $\because$ a une lacune après NIN, ce qui permet de proposer $[\mathrm{O}]$ au lieu de $(\mathrm{O})$. antonino en toutes lettres est caractéristique du groupe sud.

Ligne 6, les deux P de pater patriae sont visibles. il est inutile de les restituer.

Ligne 7 , nous voyons plutòt cos iv que cos iiii, mais ce chiffre est cependant bien difficile à lire. Nous aurions dans ce cas deux $i v$ au sud et deux au nord, tout le reste étant iiii. Ce choix nous semble avoir été de l'initiative du graveur, non de l'ordon- nateur. Celui-ci avait dû donner iiii, mais les graveurs avaient la latitude d'écrire $i v$ quand il leur semblait bon.

\section{$S X X X I$ L 42 Vagnas}

imp.t ael | hadria | no anto | nino aug | pio pp trib | pot vii | c... [ [m p] xxxi

La lecture de Thédenat est exacte, pour ce qui concerne les lettres.

Pour les interponctions, nous voyons à la ligne 1 imp. $t$ ael, et non imp.t.ael. Les autres interponctions notées par Thédenat ne sont pas vérifiables, et probablement fantaisistes : il note à la ligne 4 un point après aug, alors que dans toute la série des milliaires, il n'y a pratiquement pas de point en fin de ligne. Nous pensons que le point après imp est une initiative du graveur, qui s'est rendu compte aussitôt après que son modèle ne comportait aucune interponction.

\section{$S X X X I I I L 43$}

[ligne 1] | [ligne 2] | [ligne 3] | no aug | pio p p | trib pot | vii cos iv | m p xxxiii

La ligne 4 est bien : $\mid$ no aug $\mid$

Il faut lire cos et non gos. Le $\mathrm{G}$ de aug est différent.

\section{ÉTAT DE CONSERVATION}

\begin{tabular}{|c|c|c|c|}
\hline & & sommet & base \\
\hline 20 N XVII & Cruas & visible & enfouie \\
\hline $21 \mathrm{~N}$ XVI & Cruas & visible & enfouie \\
\hline 22 N XIV & Cruas & visible & cassée \\
\hline 25 N XII & Meysse & cassé & enfouie \\
\hline $26 \mathrm{~N}$ IX & Rochemaure & visible & enfouie \\
\hline $30 \mathrm{~N} \mathrm{IV}$ & N 540 & visible & visible \\
\hline $31 \mathrm{~S} \mathrm{X}$ & Mirabel & visible & visible \\
\hline $32 \mathrm{~s}$ XIII & St-Germain d'A. & croix & enfouie \\
\hline $34 \mathrm{~s} X X$ & Pradons & vis. et croix & enfouie \\
\hline $41 \mathrm{~S}$ XXX & Salavas & cassé & enfouie \\
\hline $42 \mathrm{~s}$ XXXI & Vagnas & brisé-croix & enfouie \\
\hline 43 S XXXIII & Barjac & cassé & enfouie \\
\hline
\end{tabular}

Nous ne donnons ici que les milliaires conservés. Nous avons très peu de données sur l'état de conservation des milliaires lus et perdus. 


\section{TABLEAU DES Ligatures}

Famille nord

$\begin{array}{lllll}\text { N XVII } & \text { L } 20 & \text { aeLIo } & \text { aNTon } & \operatorname{trIB} \\ \text { N XVI } & \text { L } 21 & & \text { aNToNIn } & \text { trIB } \\ \text { N XIV } & \text { L } 22 & & \text { aNTon } & \\ \text { N XII } & \text { L } 25 & & & \\ \text { N IX } & \text { L } 26 & \text { aeLIo } & & \text { aNToNIn } \\ \text { N VI } & \text { L } 28 & & \text { HAdr } & \\ \text { N IV } & \text { L } 30 & & \text { aNTon } & \\ \text { S X } & \text { L } 31 & & & \end{array}$

Famille sud

$\begin{array}{ll}\text { S XII/XIII } & \text { L } 32 \\ \text { S XVII } & \text { L } 33 \\ \text { S XX } & \text { L } 34 \\ \text { S XXI } & \text { L } 35 \\ \text { S XXII } & \text { L } 36 \\ \text { S XXIII } & \text { L } 37 \\ \text { S XXIV } & \text { L } 38 \\ \text { S XXX } & \text { L } 41 \\ \text { S XXXI } & \text { L } 42 \\ \text { S XXXIII } & \text { L } 43\end{array}$

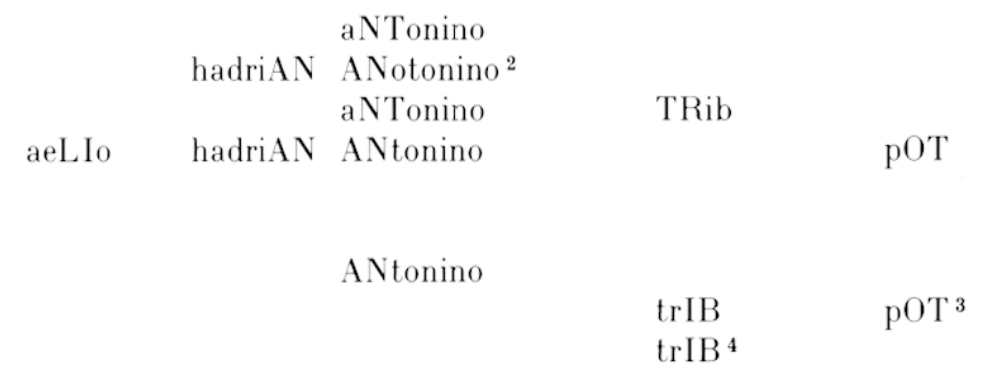

1. La ligature est bien NT et non A.V.

3. IB mal marqué, OT difficile à voir.

2. anotonino, sic.

4. IB mal marqué.

\section{HAUTEURS DES MILliaires}

Famille nord

Hauteur

N XVII

N XVI

$\mathrm{N}$ XIV

$\mathrm{N}$ XII

$\mathrm{N}$ IX

$\mathrm{N}$ VI

$\mathrm{N}$ IV

S X

Famille sud

$\mathrm{S}$ XII/XIII

S XVII

$\mathrm{S} X \mathrm{XX}$

$S$ XXI

S XXII

S XXIII

S XXIV

$S$ XXX

$\mathrm{S}$ XXXI

S XXXIII fût

L 20

L 21

L 22

L 25

L 26

L 28

L 30

L 31

$121 / 123$

$130 / 131 ; 132 \mathrm{~L}$

$101+; 100+\mathrm{L}$

$139 ; 136 \mathrm{~L}$

$122 ; 126 \mathrm{~L}$

132

$127 ; 127 \mathrm{~L}$

L 32

L 33

L 34

L 35

L 36

L 37

L 38

L 41

L 42

L 43
123

$120 ; 95 \mathrm{~L}$

4 pieds? F

$40+$ CIL

$---$

cassée

cassée

cassée

$\mathrm{L}=$ Lauxerois $; \mathrm{ms}=$ manuscrit inédit.

$\mathrm{F}=$ D. A. Flaugergues, Lettre à Séguier, 1778.

$C I L=$ Corpus Inscriptionum Lalinarum.

127 ; 127L signifie : notre mesure est de $127 \mathrm{~cm}$; celle de R. Lauxerois est de $127 \mathrm{~cm} ; 101+$ signifie $: 101 \mathrm{~cm}$ au moins, mais le monument est incomplet. 
Les hauteurs de fùts connues oscillent entre 120 et $139 \mathrm{~cm}$. On ne s'étonnera pas des minimes différences qui séparent nos mesures de celles de R. Lauxerois; il est en particulier difficile de savoir où s'arrête le fùt vers le bas.

Nous n'avons que trois mesures directes de taille globale et trois mesures anciennes. Mais comme nous savons que les bases étaient assez semblables les unes aux autres (de 43 à $53,5 \mathrm{~cm}$ ), la hauteur globale des milliaires devait osciller entre 163 et $192 \mathrm{~cm}$, ce qui représente une moyenne de 178,5 , proche des hauteurs mesurées. Notons que 6 pieds romains font $177,4 \mathrm{~cm}$.

En L 34, à Pradons, nous avons pu dégager le fùt pour mesurer sa hauteur. En plusieurs endroits, les bases actuellement enfouies fourniraient des compléments d'information intéressants.

\section{LE SOMMET DES MILLIAIRES}

Famille nord

$\begin{array}{lll}\text { N XVII } & \text { L } 20 \text { épannelé, ultérieurement poli } \\ \text { N } & \text { (sert d'accoudoir!) } \\ \text { N XI } & \text { L } 21 \text { finement épannelé } \\ \text { N XIV } & \text { L } 22 \text { grossièrement épannelé } \\ \text { N XII } & \text { L } 25 & \text {--- cassé }\end{array}$
$\mathrm{N}$ IX
$\mathrm{N}$ VI
L 26 épannelé
$\mathrm{N}$ IV
L 28 --- perdu
$\mathrm{S} \mathrm{X}$
L 30 irrégulièrement épannelé
L 31 finement épannelé, en dòme peu marqué

Famille sud

S XII/XIII L 32 --- porte le socle d'une croix

S XVII L 33 --- perdu

S XX L 34 ---épannelé - porte une croix

S XXI L 35 --- perdu

S XXII L 36 ---perdu

S XXIII L 37 ---cassé - perdu

S XXIV L 38 --- perdu

S XXX L 41 ---cassé

S XXXI L 42 ---cassé

S XXXIII L 43 ---cassé

Le hasard fait qu'on ne peut juger du sommet des milliaires de la famille sud. Pour la famille nord, le sommet est toujours épannelé, jamais poli, ce qui indique plutôt que le bloc est là encore tel qu'il était sorti de la carrière. Pour subjectifs que soient nos jugements sur l'épannelage, il semble que le travail ait été plus ou moins fin, donc que plusieurs carriers aient épannelé les blocs.

La bibliographie des milliaires ne comporte aucune indication sur leur sommet.

\section{DIAMÈTRE DES FÛTS}

Famille nord

Diamètre

$\mathrm{N}$ XVII

NXVI

$\mathrm{N}$ XIV

$\mathrm{N}$ XII

$\mathrm{N}$ IX

$\mathrm{N}$ VI

N IV

$\mathrm{S}$ X

Famille sud

S XII/XIII

S XVII

$\mathrm{S} X \mathrm{XX}$

$\mathrm{S} X X \mathrm{I}$

S XXII

S XXIII

$S$ XXIV

$S$ XXX

$S$ XXXI

S XXXIII

$\begin{array}{lll} & \text { inférieur } & \text { moyen } \\ \text { L } 20 & 51,9 & 47,5 \\ \text { L 21 } & 50,9 & 50,9 \\ \text { L 22 } & \text { perdu } & 53,2+ \\ \text { L 25 } & --- & 50,3 ; 51 \mathrm{~K} \\ \text { L 26 } & 55,7 & 49,2 \\ \text { L 28 } & --- & -- \\ \text { L 30 } & 50,3 & 50,6 \\ \text { L 31 } & 56,0 ; \text { L59,2 } & 52,7\end{array}$

$\begin{array}{ll}\text { L } 32 & 50,9 ; \mathrm{L} 49,65 \\ \text { L } 33 & --- \\ \text { L } 34 & 51,2 \\ \text { L } 35 & --- \\ \text { L } 36 & --- \\ \text { L } 37 & --- \\ \text { L } 38 & --- \\ \text { L } 41 & 41,4 \\ \text { L } 42 & 47,1 \text { usé } \\ \text { L } 43 & \text { cassé }\end{array}$

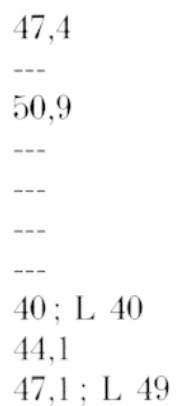

$\begin{array}{ll}\text { supérieur } & \text { ovale } \\ 45,2 ; \text { L } 45 & 45-40 \\ 49,9 ; \text { L } 50 & \text { rond } \\ 47,4 ; \text { L } 46 & 46-45 \\ 48,7 & \text { cassé } \\ 50,3 ; \text { K } 50 & 50,5-48,5 \\ -- & --- \\ 50,3 ; \text { L } 50 & \text { rond } \\ 48,5 ; \text { L } 49,3 & 47-48\end{array}$

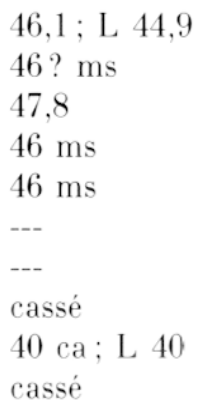

$\mathrm{L}=$ Lauxerois $: \mathrm{K}=\mathrm{König:} \mathrm{ms}=$ manuscrit.

De très faibles différences de mesures entre celles de ces auteurs et les nòtres sont normales. 
Les mesures données avec une décimale sont calculées à partir de la circonférence : on tiendra compte de la rugosité de la pierre, et du fait que le mètre souple peut être légèrement oblique. Le chiffre obtenu est de toute façon théorique, quand le milliaire est ovale.

Les chiffres donnés dans la colonne ovale donnent d'abord la mesure perpendiculaire à l'axe de l'inscription, puis la mesure axiale. Si on compare ce chiffre à la mesure directe, la géométrie n'y trouve pas toujours son compte! C'est que les ovales ne sont pas parfaits, et que les bords irréguliers et émoussés ne permettent pas toujours de bonnes mesures.

Un fût peut être de diamètre constant, avec même un renflement (L, 30). Mais la plupart vont s'amincissant vers le haut, plus ou moins régulièrement. Nous laissons aux spécialistes de la taille de la pierre le soin de dire si cet amincissement est lié à la technique de taille. Il ne nous semble pas qu'il s'agisse d'un souci esthétique, car à l'œil, les milliaires semblent cylindriques. Néanmoins, n'oublions pas qu'on a construit le Parthénon tordu pour qu'il semble droit!

\section{LA SECTION DE LA BASE DES MILLIAIRES}

Famille nord

$\begin{array}{llll} & & \text { largeur } & \text { profondeur } \\ \text { N XVII } & \text { L } 20 & 53 & 53 \\ \text { N XVI } & \text { L } 21 & 53 & 53 \\ \text { N XIV } & \text { L } 22 & \text { cassé } & \text { cassé } \\ \text { N XII } & \text { L } 25 & 51 & 51 \\ \text { N IX } & \text { L } 26 & 54 & 54 \\ \text { N VI } & \text { L } 28 & \text { perdu } & \text { perdu } \\ \text { N IV } & \text { L } 30 & 54 & 41 \\ \text { S X } & \text { L } 31 & 57,5 \text { f/57 dos } & 57 \\ \text { Famille sud } & & & \\ \text { S XII/XIII } & \text { L } 32 & 53 & \\ \text { S XVII } & \text { L } 33 & \text { perdu } & \text { perdu } \\ \text { S XX } & \text { L } 34 & \text { enfoui } & \text { enfoui } \\ \text { S XXI } & \text { L } 35 & \text { perdu } & \text { perdu } \\ \text { S XXII } & \text { L } 36 & \text { perdu } & \text { perdu } \\ \text { S XXIII } & \text { L } 37 & \text { perdu } & \text { perdu } \\ \text { S XXIV } & \text { L } 38 & \text { perdu } & \text { perdu } \\ \text { S XXX } & \text { L } 41 & 45 & 38+ \\ \text { S XXXI } & \text { L } 42 & \text { enfoui } & \text { enfoui } \\ \text { S XXXIII } & \text { L } 43 & 50 & \text { cassé }\end{array}$

Bien qu'on ait peu d'indications sur la famille sud, tous les blocs quadrangulaires semblent avoir eu approximativement la même section.

Nous ne trouvons dans la bibliographie aucune indication sur les bases.

On notera que les mesures données ne sont proches ni du pied et demi $(44,35 \mathrm{~cm})$ ni du double pied $(59,14)$.

\section{LES TRIANGLES}

Famille nord

N XVII L 20 La partie arrière de la base est irrégulière et dessine en particulier un bourrelet à l'arrière droit.

N XVI L 21 Le triangle avant droit n'est pas visible.

NXIV L 22 Perdus.

N XII L 25 Horizontaux. Épannelés.

N IX L 26 Plutòt épannelage fin que polissage.

N IV L 30 Épannelage fin.

S X L 31 Épannelage fin, les triangles sont horizontaux au lieu d'ètre en pente.

Famille sud

S XII/XIII L 32 Presque polis, mais l'épannelage commence en bas.

S XX L 34 Presque polis, mais l'épannelage commence en bas.

S XXX L 41 Épannelés. Rongés à l'arrière.

S XXXI L 42 Enfouis.

S XXXIII L 43 Le triangle avant gauche est plus grand que le droit. Les deux triangles arrière manquent.

Les triangles, ou "congés", sont les plans de transition entre le fùt cylindrique et la base carrée. Ils sont à la jonction dune partie polie, le fût, et dune partie épannelée, la base. Sauf indication contraire ou particularite, ils sont généralement épannelés, mais plus finement que la base.

Nous nous bornons aux milliaires conservés, la bibliographie ne s'étant pas intéressée aux triangles. 


\section{CORRESPONDANCES}

\section{Famille nord}

\begin{tabular}{|c|c|c|c|c|c|}
\hline & LAUXEROIS & $\begin{array}{l}\text { CIL XIII } \\
\text { ou EsPÉrandiEU }\end{array}$ & $\begin{array}{l}\text { Histoire } \\
\text { du Languedoc }\end{array}$ & KöNIG & CIL XVII \\
\hline N XVII & L 20 & 5564 & 1915 & 137 & 175 \\
\hline N XVI & L 21 & Esp. 653 & & 138 & 176 \\
\hline N XIV & L 22 & 5565 & 1913 & 139 & 177 \\
\hline N XII & L 25 & 5567 & 1912 & 141 & 179 \\
\hline N IX & L 26 & 5568 & 1910 & 142 & 180 \\
\hline N VI & L 28 & 5569 & 1909 & 144 & 182 \\
\hline N IV & L 30 & 5570 & 1908 & 145 & 183 \\
\hline S X & L 31 & Esp. 652 & & 151 & 189 \\
\hline \multicolumn{6}{|l|}{ Famille sud } \\
\hline S XIII & L 32 & 5573 & 1919 & 153 & 191 \\
\hline S XVII & L 33 & 5574 & 1920 & 154 & 192 \\
\hline S XX & L 34 & 5575 & 1921 & 155 & 193 \\
\hline S XXI & L 35 & 5576 & 1922 & 156 & 194 \\
\hline S XXII & L 36 & 5577 & 1923 & 157 & 195 \\
\hline s XXIII & L 37 & 5578 & 1924 & 158 & 196 \\
\hline S XXIV & L 38 & 5579 & 1925 & 159 & 197 \\
\hline S XXX & L 41 & 5581 & 1927 & 162 & 200 \\
\hline S XXXI & L 42 & 5582 & 1928 & 163 & 201 \\
\hline S XXXIII & L 43 & 5583 & 1929 & 164 & 202 \\
\hline
\end{tabular}

\section{NOTES DE LECTURE SUR LE CIL XVII}

Le tome XVII, 2 du Corpus Inscriptionum Latinarum, paru en 1986, n'a pu tenir compte de l'ouvrage de R. Lauxerois (paru en 1983) que nous avons utilisé, et qui représente un stade ultérieur de l'étude de ces milliaires. Nous ignorons aussi si l'auteur du CIL a passé autant de temps que nous devant chaque pierre. Nous n'avons donc pas voulu alourdir le présent article en discutant au fil du raisonnement la présentation qu'il en fait.

Cependant, comme le $C I L$ va servir immanquablement de référence, il nous parait utile de regrouper ici quelques notes de lecture.

\section{La provenance des milliaires}

\section{$175=\mathrm{L} 20=\mathrm{N}$ XVII}

On possède un renseignement de 1780 , date où le milliaire est signalé dans la crypte de l'église de Cruas. Le point $X$ XVIl était, si on nous suit, à environ $2400 \mathrm{~m}$ au nord de l'église de Cruas.

\section{$176=\mathrm{L} 21=\mathrm{N} X V \mathrm{I}$}

Ajouter à prope ecclesiam in meridiem spectans "dans le terrain Montméan".
$189=\mathrm{L} 31=\mathrm{SX}$

Le dossier du musée de Saint-Germain-en-Laye nous a permis de préciser le lieu de trouvaille : à la cote 264 (fig. 14).

$198=$ L 39

La notice ne tient pas compte des notes manuscrites d'Ollier de Marichard qu'a utilisées R. Lauxerois. Nous n'avons pas utilisé ces milliaires dont l'existence est incertaine, et pour lesquels on ignore finalement le chiffre des milles.

\section{Le déchiffrement}

175 L $20 \mathrm{~N}$ XVII

- Lire anton, non antoni (avec ligature ni)

- Pas de point en fin de ligne après hadr

- Points après aelio et ligne 4 pio. p. $p$

176 L 21 N XVI

- Le $t$ est à sa place en tête de ligne 2

- Ligne 3 antonin, pas antoni

- Pas de point ligne 2 après hadr 
- pio. p. p. trib et non pio p p. trib

- Après pot, cos et m.p, lacunes de la pierre qui peuvent permettre de restituer des points.

177 L 22 N XIII

- Le point après $t$ a été vu, mais non celui après $a u g$

- Quatre lacunes là où on attend des points, après imp, ael, pio et $p p$

\section{L 25 N XII}

- On voit quelques traces de la première ligne, dont le bas du $c$ de caes ou caesare

- Points après antonin, test, vii

180 L 26 N IX

- Lire cos. iiii, non cos iiii

183 L $30 \mathrm{~N}$ IIII

- imp. caes, non imp caes

189 L 31 S X

- Pas d'observations

\section{L 32 S XIII}

- Nous avons lu ligne 1 ---caesar, ligne 2 ---elha. A partir de la ligne 3 , on arrive à vérifier la lecture traditionnelle.

\section{L 34 S XX \\ - Pas d'observations}

\section{L 41 S XXX}

- Nous lisons plus largement -..nin [o] |aug pio $\mid$ pp trib pot | vii cos iv $\mid m p x x x$, en restituant le $o$ de antonino, car il y a une lacune de la pierre qui le permet, mais en hésitant entre cos iv et cos iiii.

\section{$201 \mathrm{~L} 42 \mathrm{~S}$ XXXI}

- Nous avons pu vérifier l'ancienne lecture de Thédenat, et même noter un point après imp, ce qui contredit la règle de l'absence complète d'interponctions dans le groupe sud, mais doit ètre une erreur de gravure aussitôt abandonnée.

\section{L 43 S XXXIII}

- Il n'y a pas de point entre $m$ et $p$

Joëlle Napoli et René Rebuffat

\section{ANNEXE I}

\section{LE MILLIAIRE DE MIRABEL}

Nous avons pu, grâce à $M^{\text {me }}$ Françoise Beck, non seulement examiner commodément au musée de Saint-Germain-en-Laye le milliaire dit de Mirabel, mais encore disposer d'un dossier possédé par le musée. Il comporte une étude inédite due à M. Grégoire qui a précisé, cadastre à l'appui, la situation de la ferme de l'inventeur du milliaire, Longin Ducros, qui a trouvé le milliaire en 1897 et l'a vendu l'année suivante au musée de Saint-Germain-en-Laye. Selon l'inventeur, le milliaire a été trouvé à $150 \mathrm{~m}$ de la ferme, dont il était séparé par un ravin.

D'autre part, d'après l'étude de M. Grégoire, passe à $100 \mathrm{~m}$ au sud de la ferme le chemin que le cadastre de 1813 appelle "grande route d'Aubenas à SaintJean-le-Centenier", cette grande route ayant été déclassée en chemin vicinal quand a été choisi l'itinéraire de la route actuelle, la Nationale 102, par Villeneuve-de-Berg et La Villedieu. A l'est de la ferme, une partie de cet ancien itinéraire garde les noms de "vieux chemins" ou "chemins romains". C'est l'ancien itinéraire de l'époque médiévale et royale, et très certainement l'ancienne voie romaine.

Au sud-ouest et à $200 \mathrm{~m}$ de la ferme, se trouve le carrefour de la cote 264 situé sur cette voie romaine, d'où se détache un ancien itinéraire vers l'est, et deux autres vers le sud (fig. 14). Ces deux itinéraires sud, l'un suivant la crête de la Costeraste, l'autre plus proche de la vallée de l'Auzon, aboutissent tous deux au village de Saint-Germain-d'Ardèche, où se trouve encore le milliaire S XII-XIII, dont le chiffre des milles est perdu.

La conclusion nous paraît évidente : le carrefour 264 est celui de la voie romaine bornée sous Antonin d'Alba à Nîmes, et de la voie franchissant les Cévennes par Pont-de-Labeaume. Le milliaire S X devait être placé à ce carrefour. En effet : 


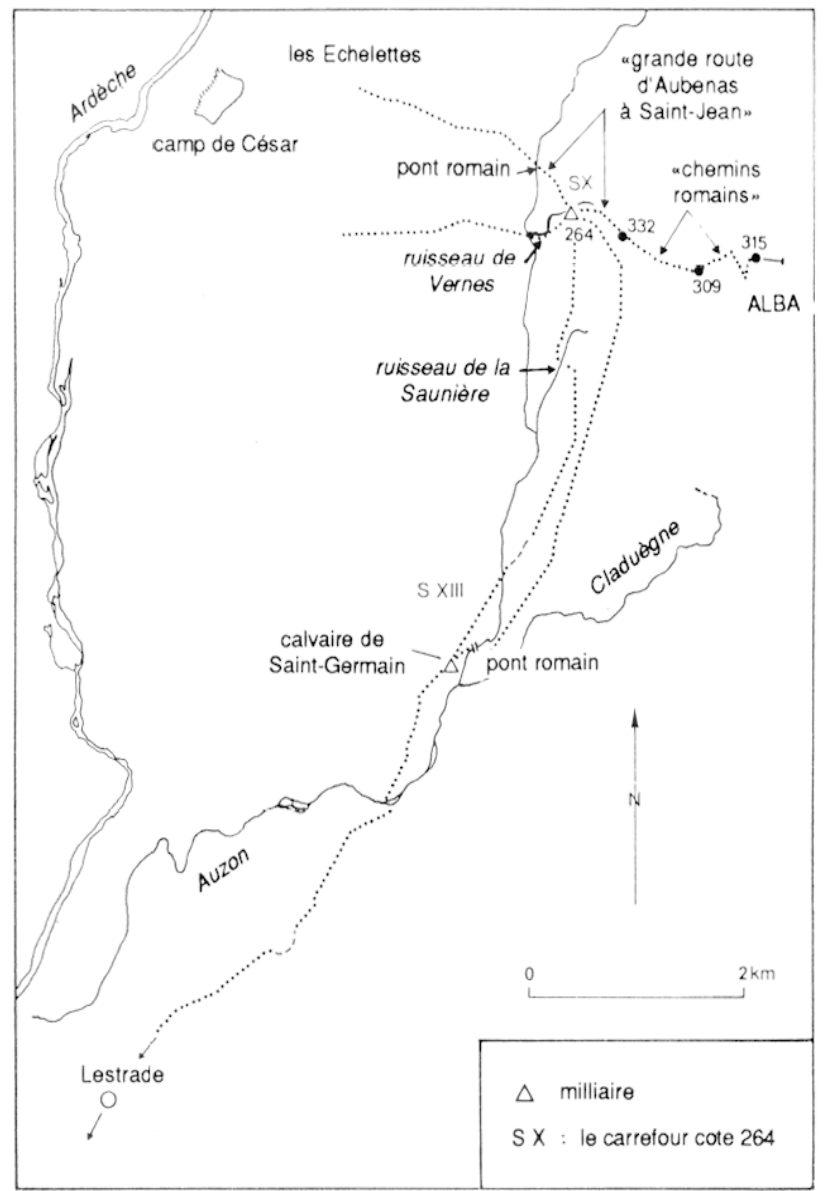

Fig. 14 - Le carrefour 264 , site du milliaire S X, et les chemins anciens de la région.

- le carrefour est à exactement $200 \mathrm{~m}$ de la ferme Longin Ducros. Comme la pierre a pu être déplacée depuis l'Antiquité, et que les $150 \mathrm{~m}$ indiqués par Longin Ducros peuvent être une simple évaluation, la précision est satisfaisante;

- le carrefour est effectivement séparé de la ferme par le ruisseau "des Vernés" (carte IGN au 1:25000) ou "de Costeraste" (cadastre), qui doit être le ravin mentionné par Longin Ducros.

La détermination de la position exacte de ce milliaire permet de noter que le milliaire-calvaire de Saint-Germain-d'Ardèche est à $4150 \mathrm{~m}$ à vol d'oiseau du carrefour 264 (fig. 14). De 264 au milliaire de Saint-Germain par le chemin de l'Auzon, la distance était de $4400 \mathrm{~m}$ environ; par la crête de Costeraste, de $4750 \mathrm{~m}$ environ. Or, deux milles font $2957 \mathrm{~m}$, trois $4435,5 \mathrm{~m}$. Le milliaire de Saint-Germain se trouve donc pratiquement exactement à trois milles romains du milliaire $\mathbf{S} \mathrm{X}$. A moins qu'on ne l'ait déplacé d'un mille romain quand on l'a utilisé comme calvaire, ce qui est bien invraisemblable, c'était donc très probablement le milliaire S XIII.

Si nous faisons l'hypothèse qu'il ait été très peu déplacé, il nous indiquerait encore le passage de la voie romaine. C'était d'ailleurs l'opinion de Thédenat, qui écrit "La voie romaine suivait l'autre côté du ravin de l'Auzon, traversait le torrent d'Auzon sur un pont dont la voûte est encore entière, et débouchait sur la route actuelle près de l'endroit où est aujourd'hui le milliaire». A partir du milliaire, la voie romaine pourrait coïncider avec la route rectiligne qui va du milliaire à l'église de Saint-Germain. 


\section{ANNEXE II}

\section{LA FRONTIÈRE NORD DE LA CITÉ D'ALBA}

Le milliaire nord XVII nous donne la limite méridionale de la zone où nous devons chercher cette frontière. Encore faudrait-il savoir où on l'a trouvé.

Deux milliaires XIV, d'Antonin et de Constantin, ont été trouvés ensemble, dans le ravin du Ferrand ${ }^{21}$, au sud de Cruas (fig. 15). On admettra aisément que la trouvaille simultanée de deux milliaires indique que l'on se trouve tout près du $14^{\mathrm{e}}$ mille de la voie ${ }^{22}$. Au surplus, deux milles plus au sud nous conduisent à $500 \mathrm{~m}$ de Meysse ${ }^{23}$, où était jadis le milliaire nord XII, et quatre milles encore au sud de Rochemaure, au voisinage sud immédiat de la Croix de la Lauze ${ }^{24}$ où était le milliaire VIIII ${ }^{25}$. Toutes ces localisations sont donc solides.

Si nous repartons du ravin du Ferrand vers le nord, nous trouvons à $2100 \mathrm{~m}$ au nord l'église abbatiale de Cruas, d'où provient le milliaire d'Antonin nord XVI qui devait être originellement à $2957 \mathrm{~m}$ du XIV : on pense que ce milliaire n'était pas en place ${ }^{26}$, et on peut admettre ce déplacement d'envi-

21 CIL, XIII, 5565 (d'après At.mer, Bulletin de la Drôme, 1878) in uia ferrata.facienda, ce qui donne une position exacte.

22 On se demande si un milliaire XV n'a pas été trouvé au même endroit (LauxreroIs, 1983, p. 255). Mais le chiffre $\mathrm{XV}$, qui n'est pas satisfaisant par rapport aux autres indications sur les lieux de trouvaille des milliaires, est à l'envers.

23 Est signalé pour la première fois dans l'ancien cimetière de Meysse : CIL, XIII, 5567 d'après Ar.r.MFr, l.c. et une copie de Thédenat.

24 Lauxrais, 1983, p. 256 : "Trouvè non loin de Rochemaure, près de la Croix de la Lauze ..."; - BLANC, 1975, p. 64, $\mathrm{n}^{\circ} 71$ : "Au quartier de la Croix de la Lauze, près du calvaire". Ce calvaire n'est pas sur la carte de l'IGN. Les six milles tombent à $500 \mathrm{~m}$ au nord du ruisseau de Chambeyrol.

25 Du sud de la Croix de la Lauze au milliaire IIII, à son emplacement actuel sur le bord de la route nationale, il y a presque exactement 4 MP. Or on peut admettre que ce milliaire IIII est quasiment en place, car il a été "trouvé dans la vallée de Mélas au pied du coteau des Combes") (CIL, citant J. Rouchifr, Histoire religieuse, civile el politique du Vivarais, $1^{\text {re }}$ éd., Paris, 1861, p. 73 ), soit un peu au nord de son emplacement actuel.

26 Lauxerois, 1983, p. 254 : "Trouvé en 1925 dans le terrain Montméan, près des murs méridionaux de l'église abbatiale. Trouvé debout, il n'était pas, croit-on, à sa place originelle". ron $900 \mathrm{~m}$. Le lieu de trouvaille du milliaire XVII est inconnu ${ }^{27}$, mais un mille de plus nous conduit approximativement entre le hameau de Corles et.le lit du Sichier, à environ $250 \mathrm{~m}$ de ce dernier. Voici donc la limite méridionale de la zone de recherche de la frontière.

Cette frontière de la cité d'Alba était-elle très loin? Si nous admettons que la route des Helviens a été bornée de frontière à frontière, il nous manque d'autant plus de milliaires que cette frontière était plus loin du Sichier. Mais plus il nous manque de milliaires, plus le fait qu'aucun ne soit conservé est invraisemblable.

Nous pouvons peut-être utiliser la démonstration que nous avons essayé de conduire plus haut. Nous avons en effet tenté de montrer que la limite fixée aux deux ateliers était un carrefour situé dix milles pas à l'ouest d'Alba. Si les deux frontières sud et nord des Helviens avaient été à peu près équidistantes d'Alba, il aurait été bien tentant de fixer la limite à la cité elle-même. Nous supposerons donc que la frontière nord des Helviens était beaucoup plus proche d'Alba par la route que la frontière sud.

En fait, c'étaient probablement les distances du carrefour-origine aux deux frontières qui étaient à peu près équivalentes (supra, p. 62-63), ce qui permet de conclure que la frontière nord était à peu près à vingt-sept milles du carrefour, soit à dix-sept milles d'Alba et à trois milles du Ferrand, ou un peu plus, mais pas beaucoup plus. La vallee de l'Eyrieux, qui est à environ $22 \mathrm{~km} / 15 \mathrm{MP}$ du ravin du Ferrand, soit à $29 \mathrm{MP}$ d'Alba, était donc au-delà de la frontière nord des Helviens.

Et de ce fait, si on peut admettre que l'Eyrieux a été vers le $x^{e} s$. la frontière nord du diocèse de Viviers ${ }^{28}$, on sait aussi qu'au sud de l'Eyrieux, l'église de Saint-Alban, au bord nord de l'Ouvèze,

27 Lauxerors, 1983, p. 253 : «Exhumé en 1849 de l'église souterraine de Cruas où il se trouvait depuis longtemps ..."; au moins depuis 1780 , d'après un manuscrit inédit. 28 LauXerois, 1983, p. 59. 


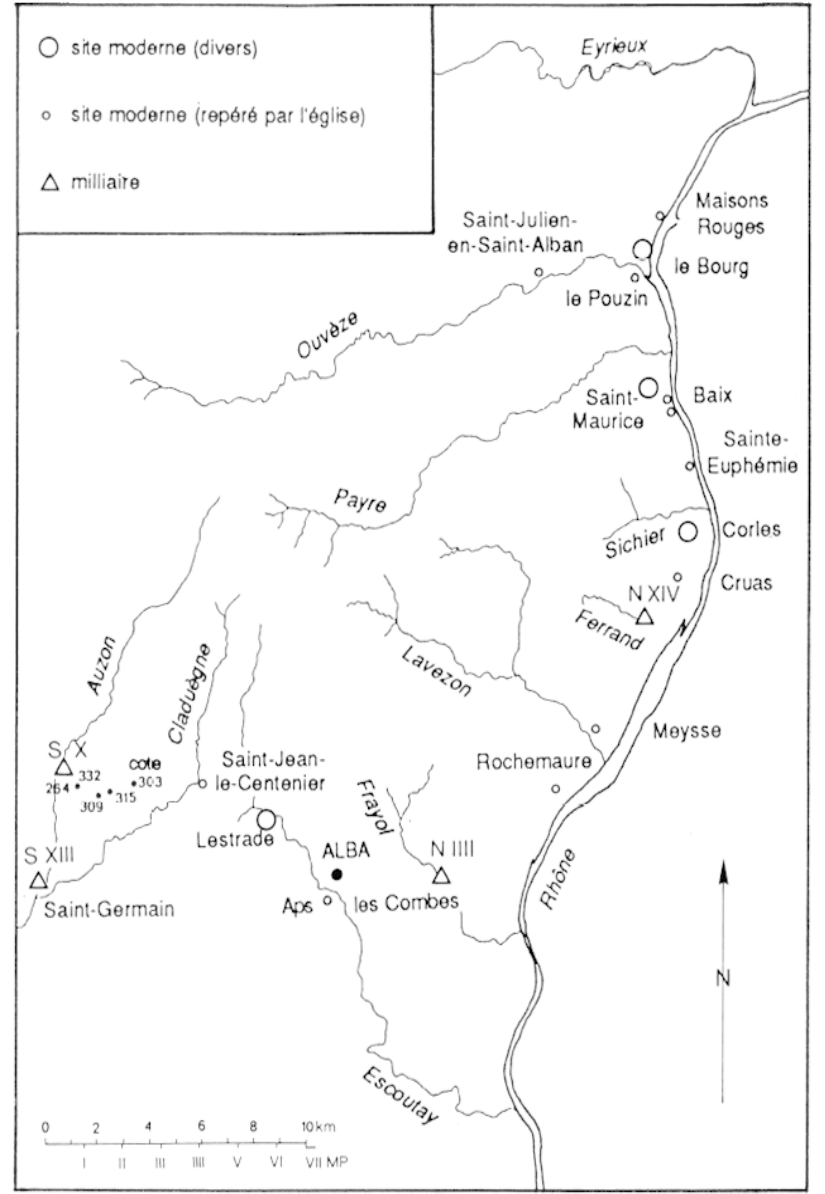

Fig. 15 - La partie nord de la route (avec quatre milliaires localisés avec précision).

faisait partie du Valentinois au $\mathrm{VI}^{\mathrm{e}}-\mathrm{v}_{\mathrm{II}}{ }^{\mathrm{e}} \mathrm{s}^{29}$. Cette indication semble bien témoigner d'un état ancien des limites. Or l'Ouvèze est à $14 \mathrm{~km} / 9.5 \mathrm{MP}$ du Ferrand, soit à 23,5 MP d'Alba. La vallée de l'Ouvèze est donc une candidate sérieuse à être rivièrefrontière.

A partir du Sichier, un milliaire (XVIII) permettrait d'atteindre la chapelle de Sainte-Euphémie; trois milliaires (XVIII-XIX-XX) permettraient d'atteindre la chapelle située au nord du village de Baix; un quatrième $(\mathrm{XXI})$ la basse plaine de la Payre; deux autres encore (XXII-XXIII) la vallée de l'Ouvèze. Six milliaires totalement disparus sans laisser le moindre souvenir, c'est déjà beaucoup, et nous n'irons pas au-delà.

A regarder la carte (fig. 15), nous serions tentés de penser que la basse plaine triangulaire de la Payre constitue une zone frontalière plus nette que l'étroite embouchure de l'Ouvèze. On remarque d'ailleurs que le lit de la Payre est une limite communale (et partiellement cantonale), tandis que l'Ouvèze n'a aucun rôle limitaire. Nous imaginerions donc assez bien que la frontière des Helviens se soit accrochée d'abord aux collines marquées par le château de Saint-Maurice, pour être ensuite éventuellement fixée au lit de la Payre. Un diplôme de Charles le Chauve en août $877^{30}$ indique d'ailleurs que Le Pouzin faisait alors partie du comté de Valence : faut-il en conclure que la rive sud de l'Ouvèze appartenait alors à ce comté, ce qui inciterait à placer au sud de l'Ouvèze, donc à la Payre, la frontière cherchée? Si cette hypothèse pouvait être adoptée, nous aurions alors perdu seulement trois ou quatre des milliaires des Helviens.

J. N et R. R. 